This item was submitted to Loughborough's Research Repository by the author.

Items in Figshare are protected by copyright, with all rights reserved, unless otherwise indicated.

\title{
A coupled hydrodynamic and particle-tracking model for full-process simulation of nonpoint source pollutants
}

PLEASE CITE THE PUBLISHED VERSION

https://doi.org/10.1016/j.envsoft.2020.104951

\section{PUBLISHER}

Elsevier BV

VERSION

AM (Accepted Manuscript)

\section{PUBLISHER STATEMENT}

This paper was accepted for publication in the journal Environmental Modelling \& Software and the definitive published version is available at https://doi.org/10.1016/j.envsoft.2020.104951.

LICENCE

CC BY-NC-ND 4.0

\section{REPOSITORY RECORD}

Jiang, Jinghua, Qiuhua Liang, Xilin Xia, and Jingming Hou. 2020. "A Coupled Hydrodynamic and Particletracking Model for Full-process Simulation of Nonpoint Source Pollutants". Loughborough University. https://hdl.handle.net/2134/13626362.v1. 


\section{A coupled hydrodynamic and particle-tracking model for full-process}

\section{simulation of nonpoint source pollutants}

\author{
Jinghua Jiang ${ }^{\mathrm{b}}$, Qiuhua Liang ${ }^{\mathrm{a}, \mathrm{b}^{*}}, \mathrm{Xilin}_{\mathrm{Xia}}^{\mathrm{b}}$, Jingming $\mathrm{Hou}^{\mathrm{a}}$
}

${ }^{\text {a }}$ State Key Laboratory of Eco-hydraulics in Northwest Arid Region of China, Xi'an University of Technology, Xi'an, China

${ }^{\mathrm{b}}$ School of Architecture, Building and Civil Engineering, Loughborough University, Loughborough, UK

\section{Abstract}

Nonpoint source (NPS) particulate pollutants are a major source of urban surface pollution. It is essential to better understand and model the dynamic process of NPS pollutants to inform the design of effective strategies for urban stormwater management and pollution control. This work presents a novel coupled hydrodynamic and particle-based model to simulate the full-process dynamics of NPS particulate pollutants from detachment, transport to deposition in urban areas. A particle-based approach is proposed to represent the physical processes of pollutant detachment and deposition. The transport of pollutant particles is simulated using a random-walk particle-tracking model, which can directly trace out the trajectories of individual particles and hence identify the pathways of pollutants. The new coupled hydrodynamic and particle-based stormwater quality model is successfully validated against several analytical and experimental test cases to demonstrate its capability in accurately simulating the full-process dynamics of NPS particulate pollutants in urban areas.

\footnotetext{
*Corresponding author: Q.Liang@lboro.ac.uk; +44 (0)150 9223526
} 


\section{Key words:}

24 Urban water management; Stormwater quality modelling; Hydrodynamic model; Pollutant wash-off; Particle-tracking; Pollutant transport

\section{Introduction}

33 Today, more than $50 \%$ of the world's population are living in urban areas (Bach et al., 2014).

34 By 2040, a further 1.1 million $\mathrm{km}^{2}$ areas will be urbanised across the world (Paresi et al., 2016).

35 Urbanisation inevitably increases population density and human activity (e.g. construction, 36 manufacturing and transportation) and grows impervious ground surfaces. This will

37 subsequently create more nonpoint source (NPS) pollutants, such as heavy metals, suspended 38 solids, minerals and nutrients (Arthur et al., 2019). When a storm event occurs, these NPS

39 pollutants will be collected by the rainwater and washed off from the urban surfaces and finally

40 transported to the downstream receiving waters, causing water quality degradation (Tsihrintzis

41 and Hamid, 1997). For example, it is recognised that majority of water body pollution failures

42 in the heavily urbanised Thames river basin are caused by the NPS pollution from road runoff 
(DEFRA, 2012). Therefore, effective control of NPS pollutants in the urban runoff is essential

44 for urban water management.

With an emphasis on working with natural processes, Sustainable drainage systems (SuDS), also known as low impact development (LID) or water sensitive urban design (WSUD) in different countries/regions of the world, consist of a series of measures and practices (e.g. swales, rain barrels and artificial wetlands) that are designed and operated to attenuate runoff and mitigate flood risk and meanwhile create other benefits including water quality enhancement through filtration, sorption and biological processes (Ahiablame et al., 2012). To design and implement efficient SuDS to effectively mitigate urban water pollution and improve water quality, it is essential to better understand the full-process dynamics of NPS pollutants during a storm event, defined as detachment from the urban surfaces, transport by the urban runoff and deposition if it occurs (Arthur et al., 2019; Baek et al., 2015; Gilbert and Clausen, 2006; Hong et al., 2016a).

In the last few decades, numerous urban stormwater quality models have been developed and applied to support runoff quality simulations. In most of these models (e.g. Stormwater Management Model (SWMM), Model for Urban Stormwater Improvement Conceptualisation (MUSIC), Soil and Water Assessment Tool (SWAT), wash-off process modelling is a key component implemented to predict runoff quality (Obropta and Kardos, 2007), representing the process of accumulated NPS pollutants being washed off by the runoff during a rainfall event. Pollutant wash-off has been widely estimated using an exponential or event mean concentration (EMC) function. In an exponential function, the amount of pollutants being washed off is assumed to be proportional to the total mass of accumulated pollutants and the runoff rate. The function contains an empirical wash-off coefficient $k$ that has no direct 66 physical meaning (Hong et al., 2016a). The EMC approach relies on estimation of EMC from substantial laboratory or field measurements (Rossman and Huber, 2016). Both methods are 
empirical and largely neglect the underlying physics of the wash-off process (Hong et al., 2016a; Sage et al., 2015).

Furthermore, the current practice commonly uses hydrological models to drive wash-off modelling. Whilst they cannot predict the dynamics of runoff and overland flow at a high spatial resolution, these hydrologically based approaches estimate the mass of washed-off pollutants and add it to the nodes (e.g. manholes) of urban drainage systems, neglecting the spatial heterogeneities of urban surfaces and their influences on pollutant distribution and dynamics ( Shaw et al., 2006; Rossman and Huber, 2016; Shaw et al., 2009). Therefore, these hydrologically based models are not able to provide the temporal and spatial specificities of pollutant distribution which are useful for better assessment and operation of SuDS.

A few attempts have been made to develop physically based models to predict the wash-off process of NPS pollutants. Shaw et al. $(2009,2006)$ proposed a physically based model to simulate the process of particulate pollutants detached by raindrops from the urban surfaces, which then travel laterally at the velocity of overland flow until settling back to the ground. However, the model neglected the pollutant detachment caused by the flow stresses and was restricted to one dimension, limiting its wider application in realistic events. Hong et al. (2016a, 2016b) coupled the Hairsine-Rose (H-R) model (Hairsine and Rose, 1992a, 1992b) with the Full Shallow-Water equations for Overland Flow (FullSWOF) model to reproduce the washoff and transport processes of pollutants on urban surfaces. The coupled model was able to simulate the full process of pollutant dynamics and effectively reflect the spatial heterogeneities of pollutant movements and distribution. However, the model solves the governing equations and predicts the flow and pollutant dynamics on a Eulerian grid-based framework that can only provide the snapshots of pollutant concentration across the simulation domain. But particulate pollutant transport is inherently a Lagrangian process and an Eulerian 
model is incapable of entailing particle trajectories to trace out the pathways of pollutants, thus only providing limited value in informing the choice and design of different types of SuDS.

The random walk particle-tracking model (PTM) developed within the Lagrangian framework provides a powerful tool for describing the motions of individual particles in a deterministic Eulerian velocity field (Liang et al., 2007). It has been widely applied in water quality modelling in a range of flow conditions from river (Weitbrecht et al., 2003), estuary (Gomez-Gesteira et al., 1999) to ocean (Olascoaga et al., 2013). Huai et al. (2019) adopted a particle random displacement model to predict the concentration of suspended sediment in an

100 open channel, with a focus on analysing the vertical profile of suspended sediment concentration in a steady vegetated flow. Sämann et al. (2019) recently introduced a PTM based transport model to predict the potential contamination paths of solutes in drainage systems during a pluvial flood event. However, the model considered only point source pollutants and neglected the wash-off process, and so is not applicable to simulate the fullprocess dynamics of NPS pollutants driven by a rainfall-flooding event in urban areas.

As a summary, most of the existing models for stormwater quality modelling suffer from various restrictions. Further research is still needed to develop alternative numerical approaches to support the modelling of full-process dynamics of NPS pollutants in urban areas during a stormwater event. Given that majority of the NPS pollutants are in the form of particulates or attached to sediments of different sizes (Arthur et al., 2019; Gilbert and Clausen,

111 2006; Hong et al., 2016a), this work aims to develop and present a new stormwater quality

112 model considers the NPS particulate pollutants directly as particles to simulate their full-

113 process dynamics. The rest of the paper is organised as follow: Section 2 introduces the new

114 model framework, which is tested and validated in Section 3; finally, brief conclusions are 115 drawn in Section 4. 


\section{Model description}

117 The proposed stormwater quality model couples together three modelling components to

118 respectively represent the ambient flow hydrodynamics, pollutant wash-off and transport

119 processes. The overall modelling framework is illustrated in Fig. 1, in which the hydrodynamic

120 model is coupled to the other two components and also provides a grid/mesh for particle

121 initiation and flow fields as necessary for the following pollutant wash-off and transport

122 modelling. The wash-off process modelling is achieved using a new particle-based approach

123 to implement the H-R model for predicting particles being washed off from the urban surfaces.

124 The new particle-based wash-off model is then coupled with a random walk PTM to trace out

125 the individual transport trajectories of suspended particles and thus identify the pathways of

126 NPS particulate pollutants.

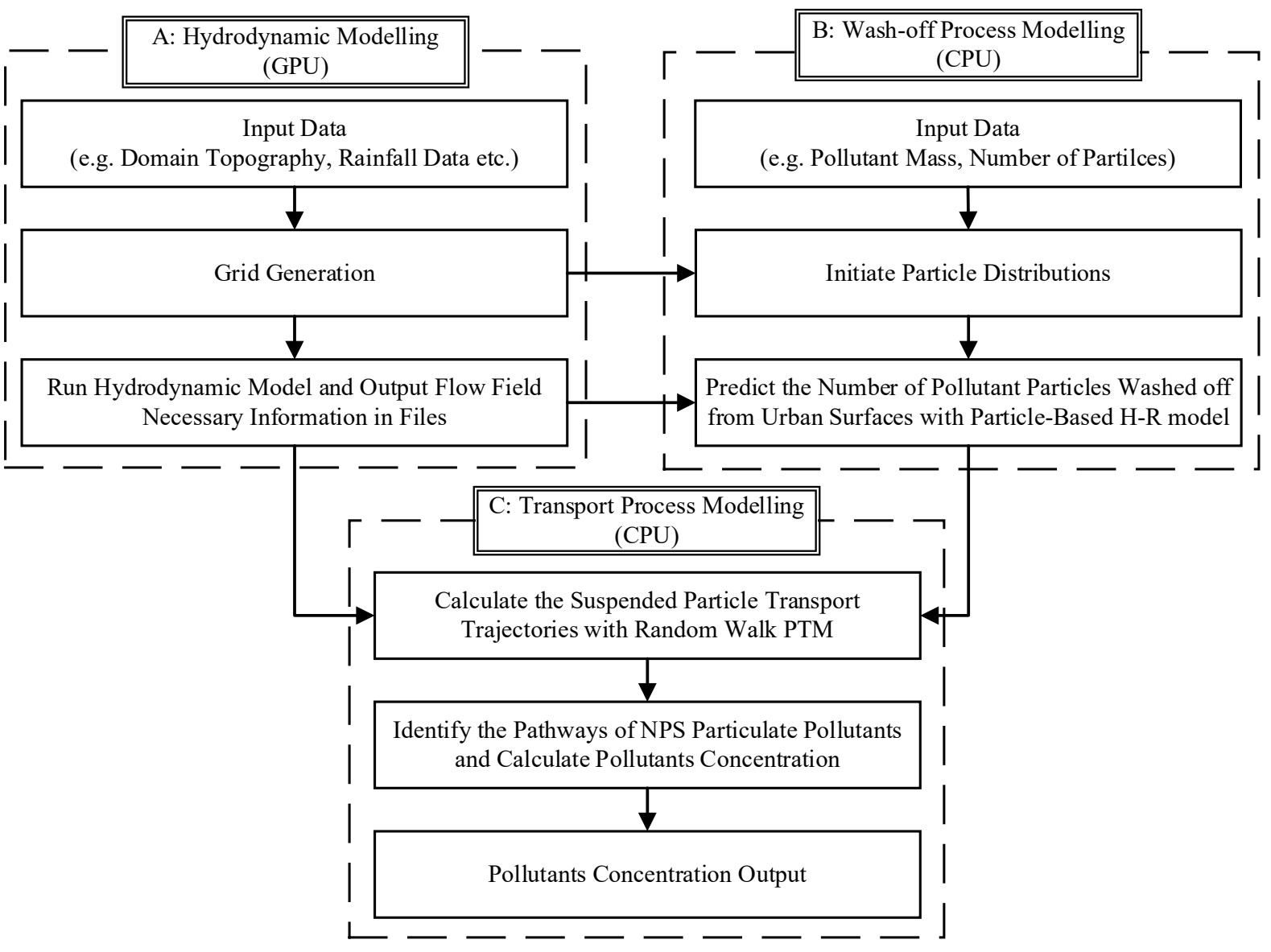




\subsection{Hydrodynamic modelling}

130 To predict the flow fields for particle tracking in the coupled stormwater quality modelling

131 framework as already shown in Fig. 1, the High-Performance Integrated hydrodynamic

132 Modelling System (HiPIMS) (Liang and Smith, 2015) is adopted in this work. HiPIMS solves

133 the full two-dimensional (2D) SWEs using a Godunov-type shock-capturing numerical scheme

134 and is implemented on multiple GPUs for substantially improved computational efficiency and

135 further technical details can be found in Xia et al. (2019). In this study, the GPU-accelerated

136 HiPIMS provides the required spatially and temporally varying flow information to drive the

137 pollutant wash-off and transport modelling components that are run on CPUs.

138

\subsection{Wash-off modelling}

139 Initially, the H-R model was coupled with a finite volume SWE model to simulate the dynamic

140 process of pollutants on urban surfaces (Hong et al., 2016a). Since a Lagrangian particle-

141 tracking model (Section 2.3) is developed and used for pollutant transport in this study, the H-

142 R model is accordingly implemented using a particle approach to describe the pollutant wash-

143 off process, which will be introduced in detail in the following sub-sections. Fig. 2. illustrates

144 the overfall particle-based framework for wash-off process modelling. In each time step, the

145 updated distribution of particles following the transport process modelling is transferred to

146 mass concentration for the subsequent wash-off estimation, i.e. quantifying the concentrations

147 of suspended and deposited pollutants. The updated mass concentration is then transferred back

148 to particle form for a new round of transport process modelling using a particle-tracking model. 


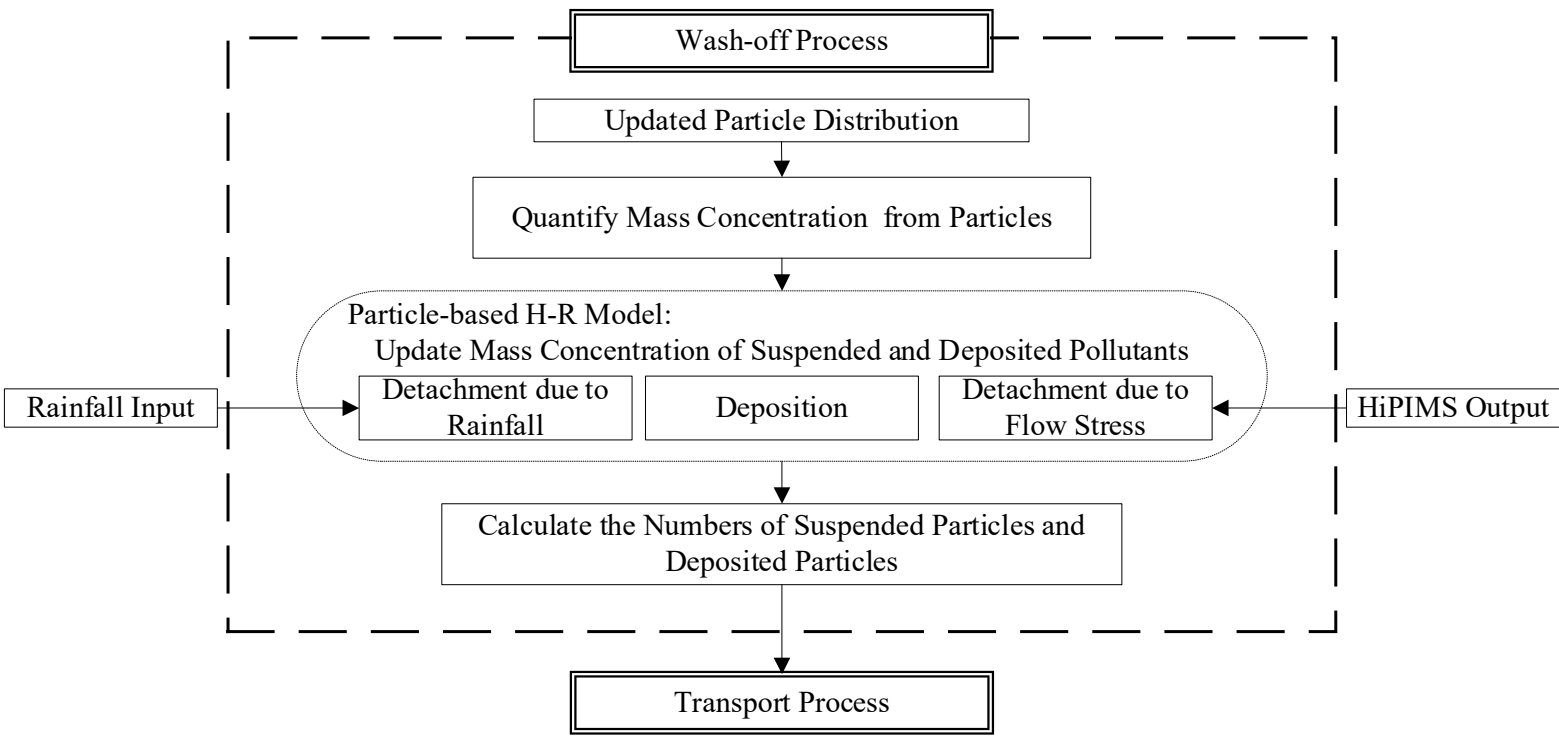

Fig. 2 The particle-based framework for wash-off process modelling.

\subsubsection{Governing equations}

152 In the proposed particle-based H-R model, the computational domain is firstly decomposed

153 into a uniform grid, which is taken to be identical to the computational grid created for

154 hydrodynamic modelling. The NPS pollutants are represented by particles of a single-class size

155 that are evenly distributed in the grid cells. During a simulation, these particles may be either

156 suspended in the flow or deposited on the ground surface. As demonstrated in Fig. 3, deposited

157 particles may be detached from the ground by rainfall impact or shear flow stress to become

158 suspended in the flow; meanwhile, the suspended particles may deposit back to the ground

159 surface if certain flow conditions are met, forming a deposition layer from which particles can

160 be re-detached again. It is noted that the model is proposed to be applied in urban areas where

161 impervious surfaces predominantly exist and therefore pollutant infiltration is not considered

162 in this work. 


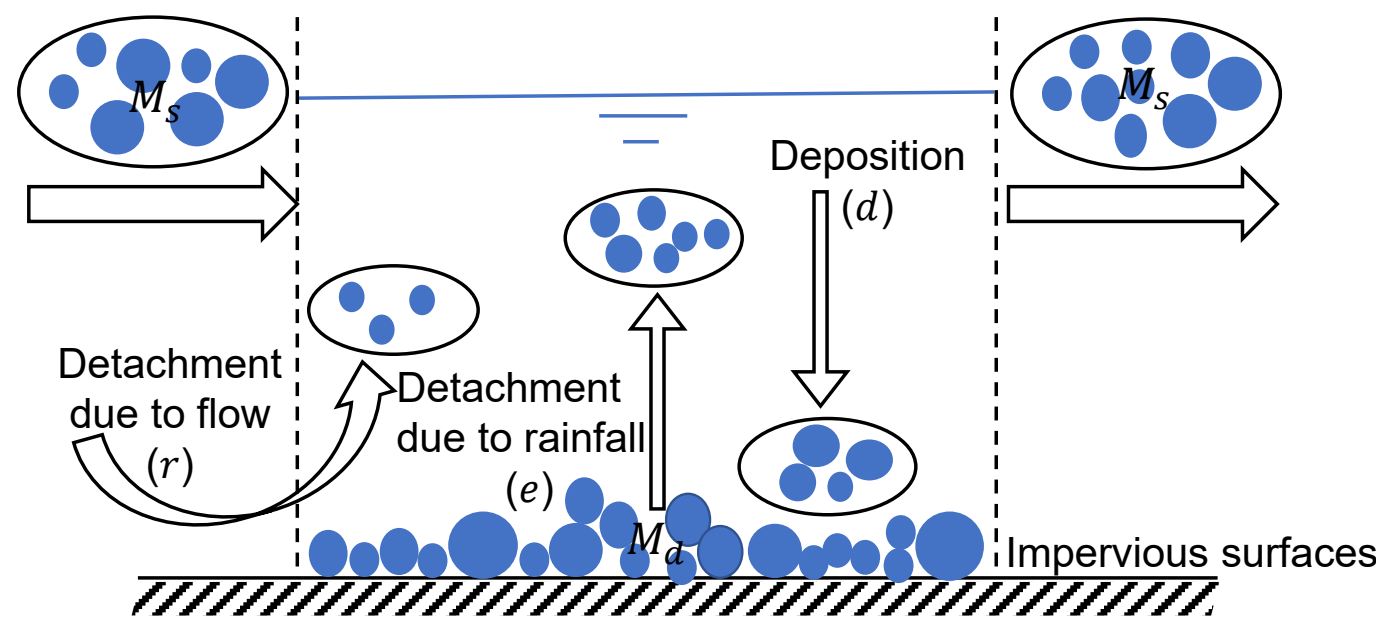

Fig. 3 The particle-based Hairsine-Rose model for pollutant wash-off.

As illustrated in Fig. 3, the particle-based mass balance equations may be written as:

$$
\frac{\partial M_{s}}{\partial t}=e+r-d
$$

$$
\frac{d M_{d}}{d t}=d-e-r
$$

where $M_{s}$ and $M_{d}$ are the mass concentrations $\left(\mathrm{kg} / \mathrm{m}^{2}\right)$ of the suspended and deposited particles, respectively; $t$ is the time (s); $e$ is the rainfall-driven detachment rate $\left(\mathrm{kg} \mathrm{m}^{-2} \mathrm{~s}^{-1}\right) ; r$ denotes the flow-driven detachment rate $\left(\mathrm{kg} \mathrm{m}^{-2} \mathrm{~s}^{-1}\right) ; d$ is the deposition rate of particles $\left(\mathrm{kg} \mathrm{m}^{-2} \mathrm{~s}^{-1}\right)$.

173 formula:

$$
e=\frac{a_{d} P M_{d}}{m^{*}}
$$

where $a_{d}$ is a coefficient representing the detachability of particles $\left(\mathrm{kg} / \mathrm{m}^{3}\right) ; P$ is the rainfall

176 intensity $(\mathrm{m} / \mathrm{s}) ; m^{*}$ is the threshold of the particle mass concentration required to shield the 
177

178

179

$$
a_{d}= \begin{cases}a_{d_{0},} & h \leq h_{0} \\ a_{d_{0}}\left(\frac{h_{0}}{h}\right)^{b}, & h>h_{0}\end{cases}
$$

in which $a_{d_{0}}$ is the initial detachability coefficient $\left(\mathrm{kg} / \mathrm{m}^{3}\right) ; h$ is the water depth (m); $h_{0}$ is the

181 threshold of water depth (m) to define the particle detachability, estimated using the following

182 formula as suggested by Mutchler and Hansen (1970):

$$
h_{0}=0.33 D_{R}
$$

where $D_{R}$ is the mean raindrop size (m).

According to Heng et al. (2011), the amount of particles required to shield the original particles from further detachment is analogous to $a_{d}$. Once the mass of particles on the ground

187 surface exceeds $m^{*}$, the deposited particles $M_{d}$ should be equal to $m^{*}$ and the threshold value $m^{*}$ may be calculated by:

$$
m^{*}= \begin{cases}m_{0}^{*}, & h \leq h_{0} \\ m_{0}^{*}\left(\frac{h_{0}}{h}\right)^{b}, & h>h_{0}\end{cases}
$$

190 where $m_{0}^{*}$ is the initial fixed threshold of mass concentration; $b$ is a positive constant (Heng et al., 2011). Accordingly, Eq. (3) can be rewritten as:

$$
e=\left\{\begin{array}{lc}
\frac{a_{d} P M_{d}}{m^{*}}, & M_{d} \leq m^{*} \\
a_{d} P, & M_{d}>m^{*}
\end{array}\right.
$$

194 particulate sediment and may be calculated using (Hong et al., 2016a): 


$$
r=\frac{\Omega_{e}}{\frac{\rho_{s}-\rho_{w}}{\rho_{s}} g h}
$$

196 where $\Omega_{e}$ is the effective stream power $\left(\mathrm{W} / \mathrm{m}^{2}\right) ; \rho_{s}$ and $\rho_{w}$ are the densities $\left(\mathrm{kg} / \mathrm{m}^{3}\right)$ of the

197 particulate sediment and water, respectively; $g=9.81 \mathrm{~m} / \mathrm{s}^{2}$ is the acceleration due to gravity.

198 The effective stream power $\Omega_{e}$ is defined as follows (Hong et al., 2016a):

$$
\Omega_{e}=F\left(\Omega-\Omega_{0}\right)
$$

200 where $F$ is the effective fraction of excess stream power; $\Omega$ represents the total stream power $201\left(\mathrm{~W} / \mathrm{m}^{2}\right)$, and $\Omega_{0}$ is the threshold of stream power $\left(\mathrm{W} / \mathrm{m}^{2}\right)$ below which no entrainment will occur. $\Omega$ is calculate by (Hong et al., 2016a):

$$
\Omega=\rho_{w} g S_{f} q
$$

where $S_{f}$ is the friction slope calculated using the Manning equation and $q$ is the water flux or unit-width discharge $\left(\mathrm{m}^{2} / \mathrm{s}\right)$.

206 The rate of particle deposition $d$ is determined by the settling velocity and available particles 207 in the flow (i.e. suspended particles):

$$
d=v_{s} * \frac{M_{s}}{h}
$$

209 where $v_{s}$ is the settling velocity $(\mathrm{m} / \mathrm{s})$ of the suspended particles, which is estimated using the 210 following equation as suggested by Cheng (1997):

$$
v_{s}=\frac{\left(\sqrt{25+1.2 d_{*}^{2}}-5\right)^{1.5} v}{d}
$$

212 where $d$ is the particle diameter $(\mathrm{m}) ; v$ denotes the kinematic viscosity $\left(\mathrm{m}^{2} / \mathrm{s}\right)$; and $d_{*}$ is given 213 by: 


$$
d_{*}=\left(\frac{\theta g}{v^{2}}\right)^{\frac{1}{3}} d
$$

215 with:

$$
\theta=\frac{\left(\rho_{s}-\rho_{w}\right)}{\rho_{w}}
$$

\subsubsection{Time step}

218 According to Heng et al. (2011), the allowable time step of the H-R model for soil erosion and

219 sediment dynamics should be restricted by the following condition:

$$
\Delta t \leq \frac{h_{\min }}{v_{s}+f(t)}
$$

where $f(t)$ is the infiltration rate of the soil. For impervious surfaces, zero infiltration rate applies, and the allowable time step may be accordingly adapted to be:

$$
\Delta t \leq \frac{h_{\min }}{v_{s}}
$$

224 However, the calculation of $e, r$ and $d$ in the H-R model is supported by the flow information 225 predicted by HiPIMS which is output at a prescribed time interval. This output time interval is commonly much larger than $\Delta t$ as defined by Eq. (16) and may subsequently cause numerical instability. To relax the restriction on the time step of the wash-off modelling, an implicit numerical scheme is adopted and implemented so that the HiPIMS output time interval can be used as the time step for wash-off calculation and the following transport process modelling.

230 The implicit time-marching scheme for the governing equations (1-2) are given as follows:

$$
M_{s}^{n+1}=M_{s}^{n}+\Delta t\left(\frac{a_{d} P M_{d}^{n+1}}{m^{*}}+\frac{\Omega_{e}}{\frac{\rho_{s}-\rho_{w}}{\rho_{s}} g h^{n}}-v_{s} * \frac{M_{s}^{n+1}}{h^{n}}\right)
$$




$$
M_{d}^{n+1}=M_{d}^{n}+\Delta t\left(v_{s} * \frac{M_{s}^{n+1}}{h^{n}}-\frac{a_{d} P M_{d}^{n+1}}{m^{*}}-\frac{\Omega_{e}}{\frac{\rho_{s}-\rho_{w}}{\rho_{s}} g h^{n}}\right)
$$

233 Herein, we re-arrange Eq. (18) and then substitute it into Eq. (17) to derive new time-marching 234 formulae for $M_{s}^{n+1}$ and $M_{d}^{n+1}$ that can be implemented explicitly:

$$
\begin{gathered}
M_{s}^{n+1}=\frac{\omega h^{n} M_{s}^{n}+(\omega-1) h M_{d}^{n}+\Delta t h^{n} \frac{\Omega_{e}}{\frac{\rho_{s}-\rho_{w}}{\rho_{s}} g h^{n}}}{\omega h^{n}+\Delta t v_{s}} \\
M_{d}^{n+1}=\frac{h^{n} M_{d}^{n}+\Delta t M_{s}^{n+1} v_{s}-\Delta t h^{n} \frac{\Omega_{e}}{\frac{\rho_{s}-\rho_{w}}{\rho_{s}} g h^{n}}}{\omega h} \\
\omega=1+\frac{a_{d}}{m^{*}} P \Delta t
\end{gathered}
$$

238 where the relevant parameters can be evaluated according to Eq. (4-14).

\subsubsection{Representing mass concentration using particles in the particle-based approach}

240 For a particle-based model as the one developed in this work, it is necessary to represent the

241 mass concentration using particles distributed in a cell for the following transport process

242 modelling. Since the total number of particles in an arbitrary cell is fixed in a single time step

243 during wash-off modelling (which is consistent with the mass balance equations (Eq. (1-2)), it

244 is only necessary to calculate the number of suspended particles and the number of deposited

245 particles can be simply obtained by subtracting the number of suspended particles from the

246 total number of particles. The mass concentration of suspended pollutants updated in Eq. (19)

247 is transferred to the number of suspended particles in the cell under consideration using the

248 following scheme:

$$
P_{s}^{i}=\frac{M_{s}^{n+1}}{m_{p}} S^{i}
$$


where $P_{s}^{i}$ is the 'number' of suspended particles in cell $i$, which is returned directly as a real

251 number; $m_{p}$ is the mass of a single particle $(\mathrm{kg}) ; S^{i}$ is the area $\left(\mathrm{m}^{2}\right)$ of cell $i$.

252 The real number $P_{s}^{i}$ calculated in Eq. (22) is then rounded to become an integer $\left(N_{s}^{i}\right)$ to 253 specify the final number of suspended particles in cell $i$ :

$$
N_{s}^{i}=\left\lfloor P_{n s}^{i}\right\rfloor
$$

255 Apparently, small mass error exists in this round-off process, which must be properly avoided.

256 Herein, de is introduced to define the remaining decimal error between $P_{s}^{i}$ and $N_{s}^{i}$ :

$$
d e=P_{s}^{i}-N_{s}^{i}
$$

258 where $d e$ is obviously always smaller than ' 1 '. To eliminate the mass error during a simulation, $259 d e$ is added up across the grid cells in sequence. When the accumulated error becomes equal 260 to or larger than ' 1 ', a suspended particle is created and placed in the current cell. The 261 subsequent remaining error will then be added to the next cell and the procedure continues until 262 all of the cells in the domain are swept through. This final decimal error will be neglected. It 263 should be noted that this procedure is implemented to largely ensure mass conservation and 264 will not create any major disturbance to the numerical solution as the mass of a particle is 265 always very small.

266 Now, the number of suspended particles in cell $i$ is updated for the next step of transport 267 process modelling and the difference between the updated $\left(N_{S}^{n+1}\right)$ and the existing $\left(N_{s}^{n}\right)$ 268 particle numbers can be readily sought:

$$
\Delta N_{s}^{i}=N_{s}^{n+1}-N_{s}^{n}
$$


270 If $\Delta N_{s}^{i}>0$, it indicates $\Delta N_{s}^{i}$ deposited particles are disturbed and become suspended from the

271 ground surface, which will be randomly distributed inside cell $i$. On the other hand, if $\Delta N_{s}^{i}<$

$2720, \Delta N_{s}^{i}$ currently suspended particles are randomly selected and become deposited.

\subsection{Particle transport modelling}

274 The suspended particles updated in the last section will be moved and transported by the urban

275 surface runoff induced by rainfall using a particle-tracking model as introduced in this section.

\subsubsection{Governing equations}

277 Since the particulate pollutants are small and their presence will not impose any accountable

278 feedback to the ambient flow hydrodynamics in a macro-scale simulation, the pollutant particles are assumed to immediately react to and move according to the flow velocity at the location where the particle is located. In a $2 \mathrm{D}$ simulation domain as considered in this work, the semi-discretised governing equations for the particle-tracking model are given by:

$$
x(t+\Delta t)=x(t)+u(x, y, t) \cdot \Delta t+\sqrt{2 D_{x x} \Delta t} \cdot \Lambda
$$

in which $(x, y)$ is the position of the particle in the phase space, $u$ and $v$ are the depth-average flow velocity predicted by the hydrodynamic model as briefly introduced in Section 2.1. The random walk terms are also included to account for the diffusion effect, with $D_{x x}$ and $D_{y y}$ respectively denoting the longitudinal and transverse diffusion coefficients $\left(\mathrm{m}^{2} / \mathrm{s}\right)$ and $\Lambda$ being a random number that conforms to a normal distribution with a 0 mean and standard deviation

289 (Follett et al., 2016; Huai et al., 2019). Herein, $\Lambda$ is generated by a random number generator in the numerical solution.

\subsubsection{Numerical scheme}


292 The governing equations (26-27) are divided into two parts to account respectively for particle

293 advection and diffusion and handled separately in the adopted numerical solution scheme. For

294 particle advection, a second-order Runge-Kutta (RK) integration method is used to update the

295 particle positions to the next time step. Taking the $x$-direction Eq. (26) as an example, the RK

296 integration scheme may be expressed as:

$$
x(t+\Delta t)=x(t)+\frac{\Delta t}{2}\left(K_{1}+K_{2}\right)
$$

where $K_{1}$ and $K_{2}$ are the Runge-Kutta coefficients defined as follows:

$$
K_{1}=u(x(t), y(t), t), \quad K_{2}=u(x(t+\Delta t), y(t+\Delta t), t)
$$

300 The advection part of the $y$-direction Eq. (27) is solved similarly.

301 It should be noted that the flow velocity fields output from HiPIMS are grid-based, i.e. $u$ and $v$ are only discretely available at the centre of each of the grid cells. A bilinear interpolation scheme is therefore implemented to obtain the flow velocities at the specific position where the particle under consideration is located (which can be anywhere within a cell) and support the above particle advection calculation.

306 Finally, for each of the particles, the random walk diffusion terms, $\sqrt{2 D_{x x} \Delta t} \cdot \Lambda$ and

$307 \sqrt{2 D_{y y} \Delta t} \cdot \Lambda$, are calculated and the resulting particle displacements are directly added to $308 x(t+\Delta t)$ and $y(t+\Delta t)$ updated by the above Runge-Kutta advection step to obtain the final 309 particle position for this time step.

\section{Results and discussion}

311 In this section, the new coupled hydrodynamic and particle-tracking model for stormwater

312 quality modelling is validated by simulating four test cases. 
314 The first test case considers the advection and diffusion of a solute cloud in a steady uniform

315 flow (Zhang et al., 2015), aiming to validate the particle-tracking model for pollutant transport.

316 In an $800 \mathrm{~m} \times 200 \mathrm{~m}$ frictionless channel with a horizontal bed, a solute cloud moves from

317 upstream to downstream, driven by a steady flow with $h=1 \mathrm{~m}, u=1 \mathrm{~m} / \mathrm{s}, v=0 \mathrm{~m} / \mathrm{s}$. The

318 analytical solution of the solute concentration $(c)$ for this idealised case is provided as:

$$
c(x, y, t)=\frac{c_{0} / h}{4 \pi t \sqrt{D_{x x} D_{y y}}} e^{\left(-\frac{\left(x-x_{0}-u t\right)^{2}}{4 D_{x x} t}-\frac{\left(y-y_{0}\right)^{2}}{4 D_{y y} t}\right)}
$$

320 with $c_{0}=233.06, D x x=1.02 \mathrm{~m}^{2} / \mathrm{s}$ and $D y y=0.094 \mathrm{~m}^{2} / \mathrm{s}$ as adopted in Zhang et al. (2015).

321 At the start of the simulation, the peak concentration is located at $\left(x_{0}, y_{0}\right)=(0,100)$. The 322 simulation starts at $t=60 \mathrm{~s}$ and the initial contours of the solute concentration as defined by 323 Eq. (30) is shown in Fig. 4. Transmissive conditions are imposed in all four lateral domain 324 boundaries. Simulation is run until $t=600 \mathrm{~s}$ with 480,000 particles while each particle 325 represents $4.86 \times 10^{-4} \mathrm{~kg}$ of mass.

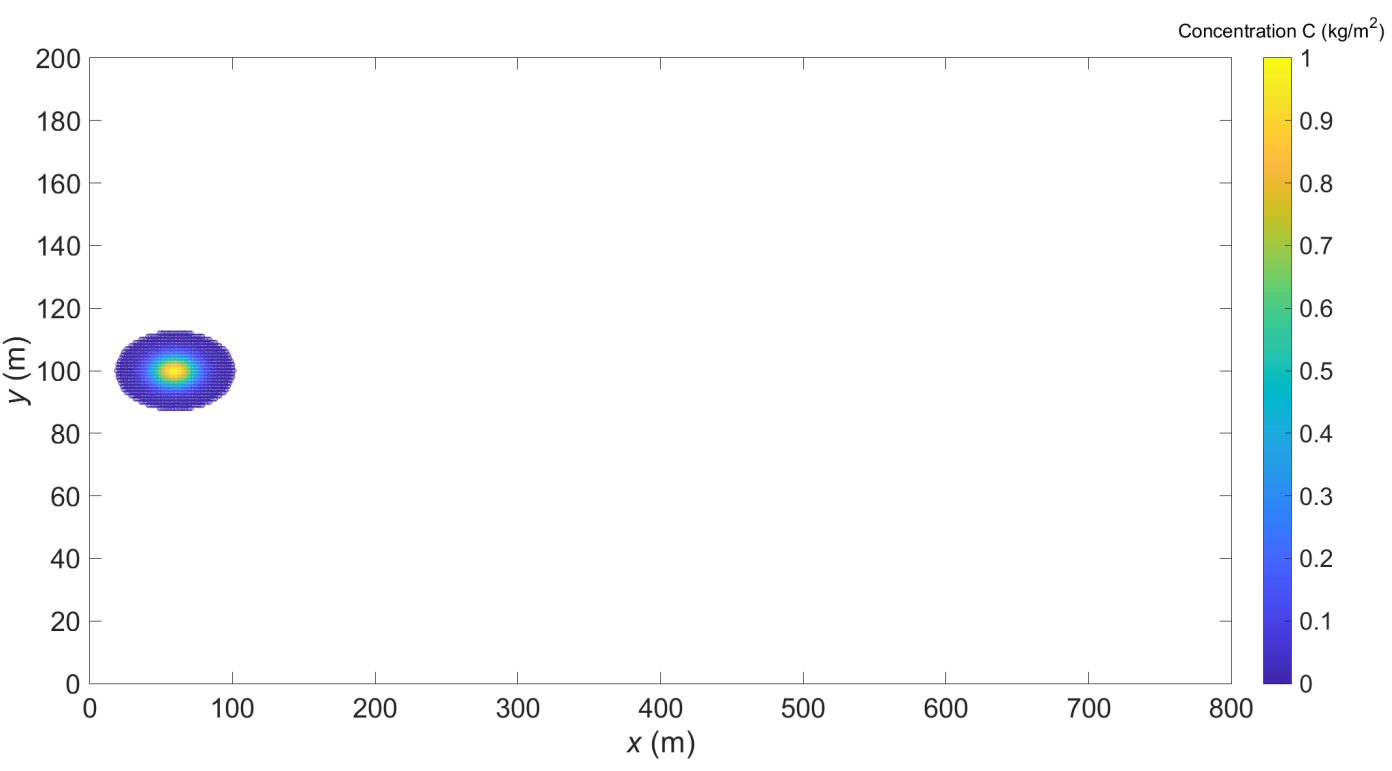

Fig. 4 Schematic of the solute cloud test at the initial time. 
Fig. 5 compares the analytical solution with the numerical prediction in terms of concentration profile at different output times. As expected, the solute cloud is driven by the

330 steady flow and transported from upstream to downstream, accompanied by the diffusion of

331 the concentration as time increases. The numerical prediction agrees satisfactorily with the 332 analytical solution, and both of the transport and diffusion processes are well captured by the 333 particle-tracking transport model.

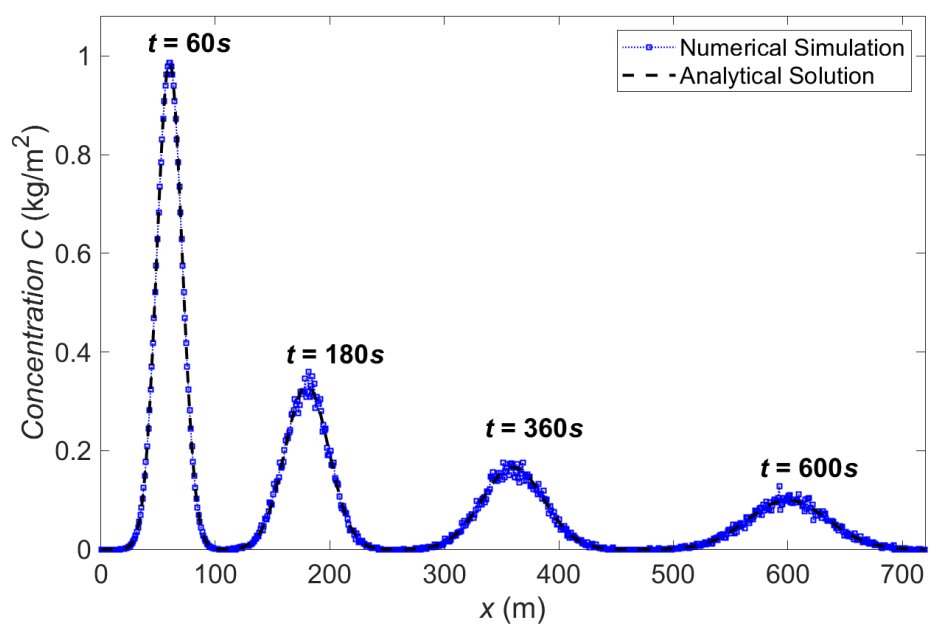

Fig. 5 Comparison between the predicted concentration profile and analytical solution.

336 In order to visualize the movement of particles, positions of all particles at different output

337 times are presented in Fig. 6 and particles are plotted in different colours according to the 338 particle density in the corresponding cell containing them. Contours of the analytical 339 concentration are also plotted in the figure for comparison. Since the solute concentration is 340 relevant to the particle density in a cell, the consistency between the particle density and the 341 analytical concentration field (as reflected by the contour lines) confirms again the capacity of 342 the current particle-tracking transport model in accurately simulating the transport of a 343 concentration field with diffusion. 


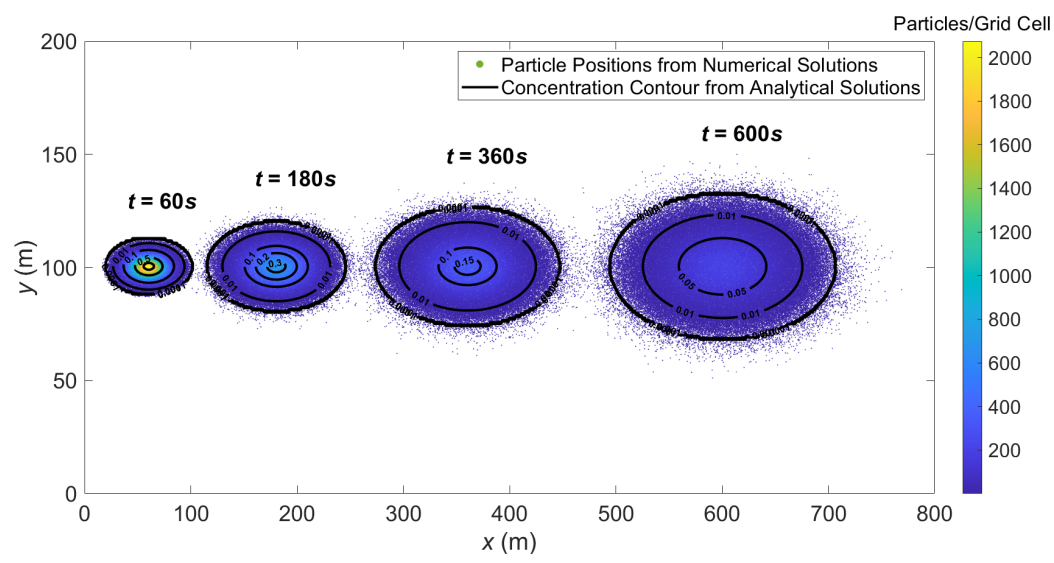

345 Fig. 6 Particle clouds and analytical concentration contours at different output times.

346 One of the main advantages of the adopted particle-based approach is its capability to trace

347 out an individual particle's trajectory to effectively depict its source, pathway and final

348 destination. Fig. 7 provides the trajectories of four sample particles in the simulation domain

349 during the $600 \mathrm{~s}$ of simulation, plotted at a $5 \mathrm{~s}$ time interval. This clearly shows each of the

350 individual particles being driven from their initial location to downstream during the simulation.

351 The diffusion effect imposed by the random walk algorithm on the particle dynamics is evident

352 as all of the particles are drifted back and forth in the $y$-direction in addition to being

353 predominantly transported by the unidirectional flow from upstream to downstream.

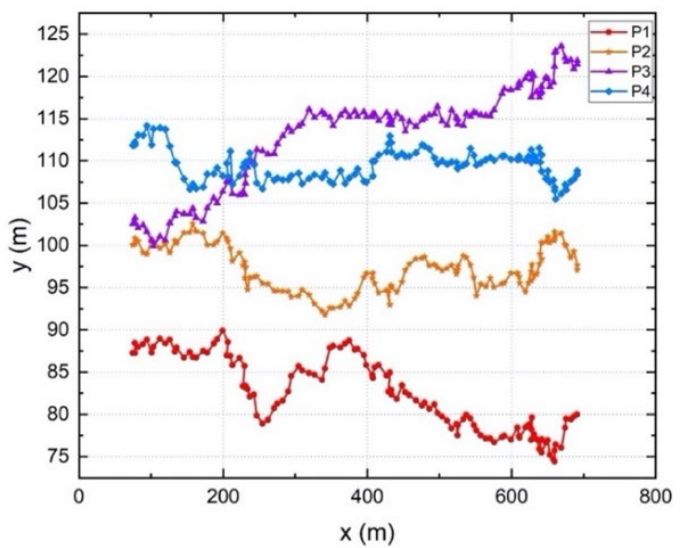

Fig. 7 Trajectories of the four selected sample particles. 


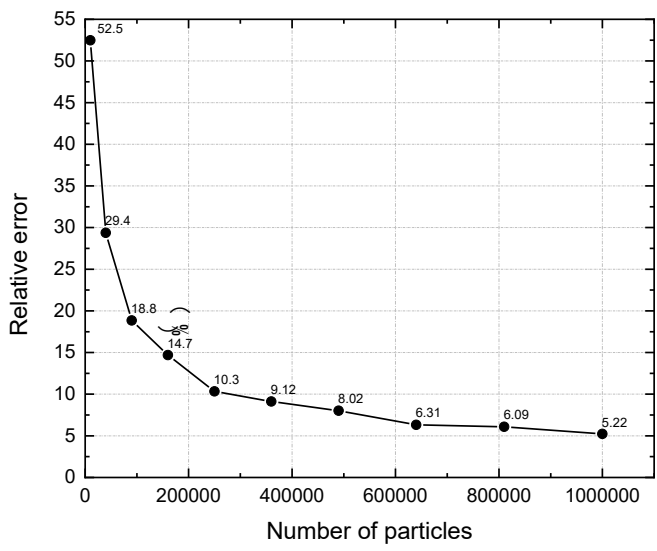

357 Fig. 8 Relative errors of the simulation results against the number of particles being used.

358 Further simulations are carried out to investigate the convergence of the simulation results

359 against the number of particles being used to represent the concentration field, ranging from

3601,000 to $1,000,000$. To quantitively compare the numerical and analytical results, the relative

361 errors are calculated and presented in Fig. 8. Apparently, the simulation errors decrease with

362 the increasing number of particles being used, which effectively indicates the convergence of

363 the simulation results. Converged simulation results are achieved from using 250,000 particles

364 and further increase of the particle number will no longer substantially improve the simulation

365 accuracy. When 1 million particles are used, there is still about $5 \%$ of relative simulation error,

366 which may be due to numerical diffusion and also the inherent error created by the normal

367 distribution based random number generation algorithm in the $\mathrm{C}++$ library (to generate the

368 random numbers in the modelling approaches as introduced in Section 2) and cannot be entirely

369 eliminated.

\subsection{Pollutant wash-off and transport in a uniform flow field}

371 Based on the previous test case as considered in Section 3.1, two more tests are designed herein

372 with different initial conditions to validate the detachment and deposition modules of the

373 proposed particle-based wash-off model. All simulations are run for $600 \mathrm{~s}$ with 250,000

374 particles. 


\subsubsection{Particle deposition during the transport process}

376 In this test, all of particles are assumed to be initially suspended in the shallow flow, i.e. no

377 pollutant particle is found on the ground surface at the start, which may be mathematically

378 expressed as:

$$
M_{d}^{0}=0
$$

$$
M_{s}^{0}=h \frac{c_{0} / h}{240 \pi \sqrt{D_{x x} D_{y y}}} e^{\left(-\frac{\left(x-x_{0}-60 u\right)^{2}}{240 D_{x x}}-\frac{\left(y-y_{0}\right)^{2}}{240 D_{y y}}\right)}
$$

381 When the simulation starts, particles are assumed to deposit at a constant settling velocity $\left(v_{s}\right)$

382 of $0.01 \mathrm{~m} / \mathrm{s}$ and no particle is allowed to re-suspend from the ground surface, i.e. the rainfall

383 intensity $(P)$ and effective fraction of excess stream power $(F)$ are both assumed to be 0 to

384 ensure zero particle detachment rates. Based on these assumptions, the analytical solution of

385 the concentration field is derived and given by:

386

$$
c(x, y, t)=e^{-0.01 t+\ln \left(\frac{c_{0}}{240 \pi \sqrt{D_{x x} D_{y y}}} \times e^{-\frac{\left(x-x_{0}-t-60\right)^{2}}{240 D_{x x}}-\frac{\left(y-y_{0}\right)^{2}}{240 D y y}}\right)}
$$

387 Fig. 9 illustrates the predicted concentration profiles output at every $100 \mathrm{~s}$. As the time passes,

388 the pollutant concentration in the flow decreases due to deposition, which is accurately 389 captured by the model. The predicted profiles compare excellently well with the analytical 390 solutions. This effectively confirms the model's performance in predicting particle deposition.

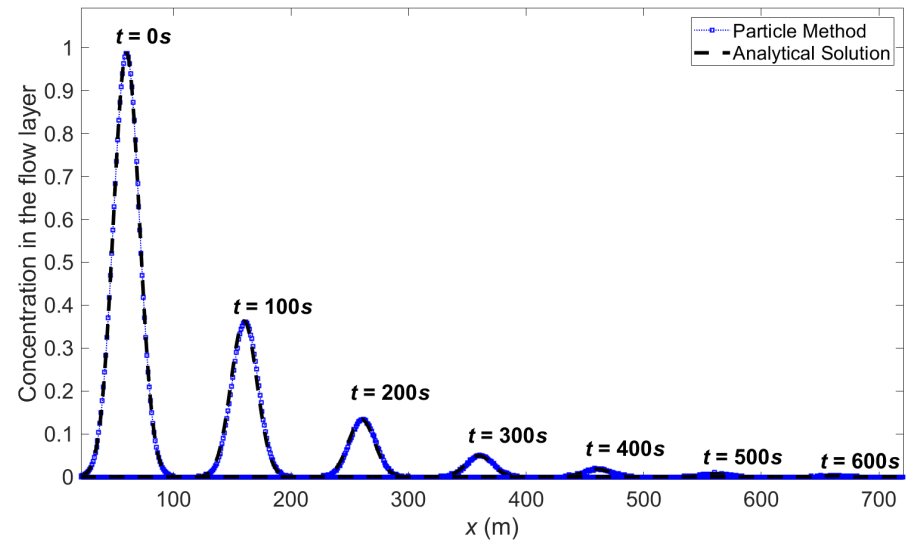


Fig. 9 Particle deposition test: numerical and analytical concentration profiles at different output times.

\subsubsection{Particle detachment during the transport process}

395 In this test, all of particles are initially attached to the ground surface, i.e. no pollutant particle

396 is found in the shallow flow at the start, which may be mathematically described as:

$$
M_{d}^{0}=\frac{c_{0} / h}{240 \pi \sqrt{D_{x x} D_{y y}}} e^{\left(-\frac{\left(x-x_{0}-60 u\right)^{2}}{240 D_{x x}}-\frac{\left(y-y_{0}\right)^{2}}{240 D_{y y}}\right)}
$$

$$
M_{S}^{0}=0
$$

399 When the simulation starts, particles are assumed to be detached from the ground surface at a

400 constant detachment rate of 0.01 and no particle will deposit back to the ground surface (i.e. $v_{s}$ $401=0$ ). To maintain the constant detachment rate, $m_{0}^{*}$ is taken to be equal to the concentration of 402 deposited particles, and $P, a_{d_{0}}, b, F$ and $D_{R}$ are set to be $0.01,1,1,0$ and 10, respectively. 403 Based on these assumptions, the analytical solution of the concentration field is given by:

$$
c(x, y, t)=e^{-0.01 t+\ln \left(\frac{c_{0}}{240 \pi \sqrt{D_{x x} D_{y y}}} \times e^{-\frac{\left(x-x_{0}-t-60\right)^{2}}{240 D_{x x}}-\frac{\left(y-y_{0}\right)^{2}}{240 D y y}}\right)}
$$

The numerical and analytical concentration profiles on the ground surface are shown in Fig.

40610 at different output times. The pollutant concentration at the initial location decreases with

407 time as the pollutant particles detached from the ground surface are transported to the 408 downstream. Excellent agreement between the numerical prediction and analytical solution 409 validates the particle detachment module of the wash-off model. 


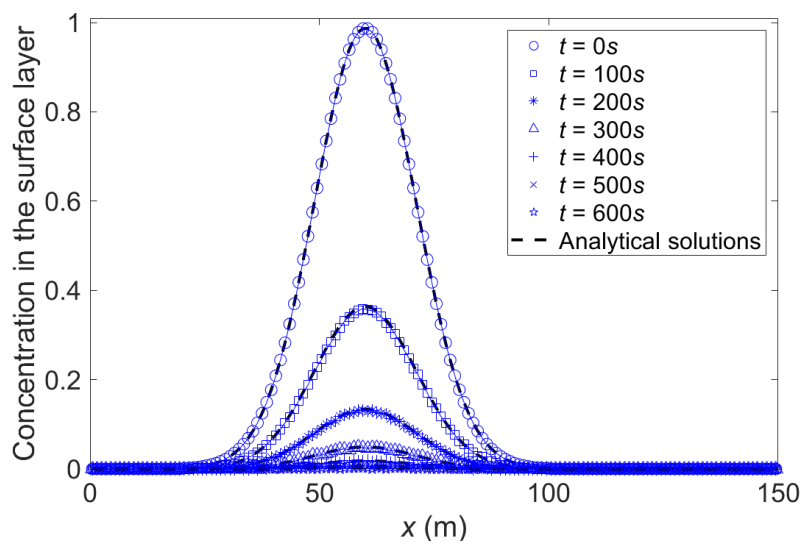

411 Fig. 10 Particle detachment test: numerical and analytical concentration profiles at different output times.

\section{$413 \quad 3.3$ Pollutant wash-off and transport on urban road surfaces}

414 In this section, the new coupled hydrodynamic and particle-based stormwater quality model is

415 further validated by reproducing the wash-off experiments reported by Egodawatta et al. (2007).

416 The experiments were designed to investigate pollutant wash-off process using simulated

417 rainfall on three urban road surfaces in the Gold Coast Region, Queensland State, Australia.

418 All three sites are located in the typical urban residential areas with different slopes. Each of 419 the sites were demarcated into even plot surfaces of $3 \mathrm{~m}^{2}(2 \mathrm{~m} \times 1.5 \mathrm{~m})$. The initial pollutant 420 loads on the road surfaces were determined by collecting samples using a vacuum cleaner from 421 the most downstream plot at each of the sites. The road names, slopes and initial pollutant loads 422 of all of the three experimental sites are summarised in Table 1.

423 Table 1 Basic information about each of the experiment sites from Egodawatta et al. (2007).

\begin{tabular}{ccc}
\hline Site & $\begin{array}{c}\text { Slope of the } \\
\text { road (\%) }\end{array}$ & $\begin{array}{c}\text { Pollutant mass on the } 3 \mathrm{~m}^{2} \\
\text { experiment sites }(\mathrm{g})\end{array}$ \\
\hline Lauder Ct. & 10 & 9.3 \\
Gumbeel Ct. & 7.2 & 32.6
\end{tabular}


Piccadilly Pl. $\quad 10.8 \quad 10.6$

During the experiments, a rainfall simulator was used to produce different rainfall intensities and apply over different plots in each of the road sites, starting from the second most downstream plot and moving upstream in sequence. The runoff samples were collected using a catch tray and a vacuum system at the downstream end of each of these plots at a given time period. The fraction wash-off $(F)$ was then defined as the weight ratio between the cumulative washed-off pollutants and the initial pollutant load. Here in this work, the experiments conducted with rainfall intensities of $40 \mathrm{~mm} / \mathrm{h}, 65 \mathrm{~mm} / \mathrm{h}, 86 \mathrm{~mm} / \mathrm{h}, 115 \mathrm{~mm} / \mathrm{h}$ and $133 \mathrm{~mm} / \mathrm{h}$ are considered and simulated to provide representative results. It should be noted that $310 \mu \mathrm{m}$ median particle size is applied in all of the three sites in this study since our numerical experiments show that the use of slightly different particle size between $305 \mu \mathrm{m}$ and $310 \mu \mathrm{m}$ (values extracted from the original median particle size gradation curve) has little impact on the final results.

437 Given the relatively small plot area and predominant convective dynamics of the rainfallinduced surface flow in these experiments, the diffusion effect in the pollutant transport process is negligible. With respect to other key model parameters in wash-off modelling, Hong et al.

440 (2016a) suggested that their values could be selected from the test ranges suggested in the 441 previous soil erosion studies (Beuselinck et al., 2002; Heng et al., 2011; Jomaa et al., 2010; Proffitt et al., 1991). Hong et al. (2019) also indicated that simulation results were not sensitive to the choices of parameters $b, F$ and $\Omega_{0}$. Therefore, these parameters directly take the median

444 values of the suggested test ranges as shown in Table 2. However, two more parameters, i.e. $445 a_{d_{0}}$ and $m_{0}^{*}$, are still needed to be specified. According to the Hong et al. $(2019,2016 \mathrm{a}), a_{d_{0}}$ 446 may be selected from a range between 1500 and $4500 \mathrm{~kg} / \mathrm{m}^{3}$ but careful calibration is needed 447 to decide an appropriate value in relation to rainfall intensity. Heng et al. (2011) suggested a 
range of values for $m_{0}^{*}$ in the context of soil erosion with infiltration effect, which is not directly applicable to the current study that is focused more on impervious urban surfaces.

Table 2 Recommended values for model parameters.

\begin{tabular}{cccc}
\hline Symbol & Units & Range & Value \\
\hline$b$ & - & $0.8-2$ & 1 \\
$F$ & - & $0.01-0.03$ & 0.02 \\
$\Omega_{0}$ & $\mathrm{~W} / \mathrm{m}^{2}$ & $0.15-0.35$ & 0.25 \\
$D_{R}$ & $\mathrm{~mm}$ & & 2
\end{tabular}

451

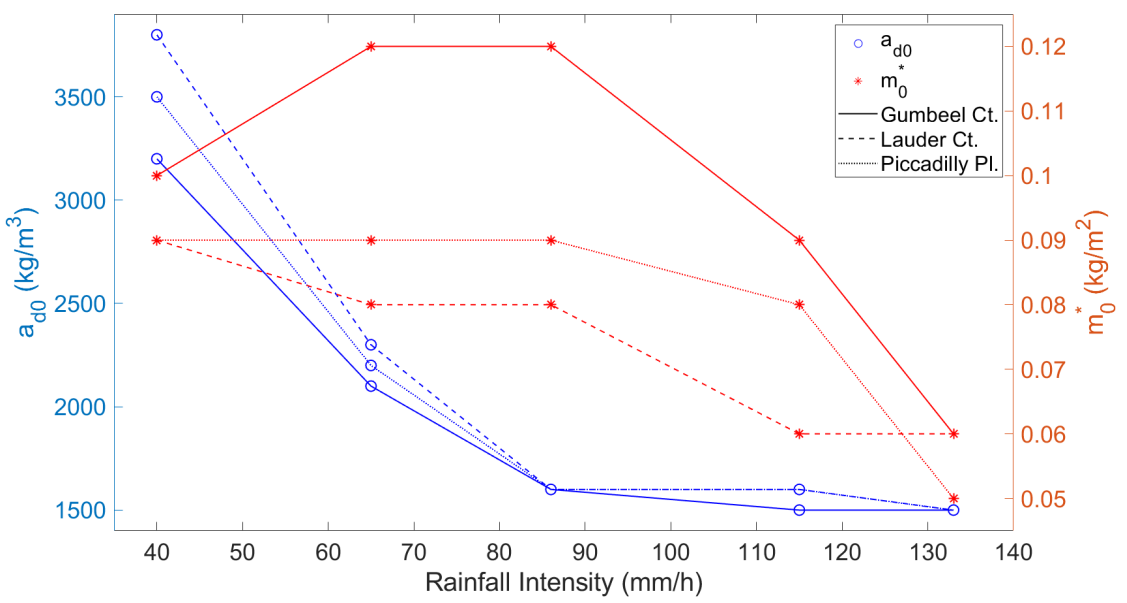

Fig. 11. Changes of model parameters $a_{d_{0}}$ and $m_{0}^{*}$ against the rainfall intensity.

To calibrate the wash-off model and find the best-fitted values for $a_{d_{0}}$ and $m_{0}^{*}$, the Nash-

Sutcliffe efficiency (NSE) coefficient and root-mean-square deviation (RMSD) are calculated by comparing the numerical simulation results with observations. The best-fitted $a_{d_{0}}$ and $m_{0}^{*}$ selected following a series of numerical experiments are listed in Table 3 and also plotted in 458 Fig. 11 against the rainfall intensity. It is evident that the best-fitted values of $a_{d_{0}}$ are consistent across the three road sites and only varies slightly for the same rainfall intensity. But $a_{d_{0}}$ is 460 found to be more sensitive to rainfall intensity and its values decrease as the rainfall intensity 
461 increases. These conclusions are generally consistent with those reposted in the previous study

462 (Hong et al., 2019) where $a_{d_{0}}$ was recommended to take the value of $3625.3 \mathrm{~kg} / \mathrm{m}^{3}$ for light

463 rain, $1523.7 \mathrm{~kg} / \mathrm{m}^{3}$ for moderate rain and $1641.9 \mathrm{~kg} / \mathrm{m}^{3}$ for heavy rain. For $m_{0}^{*}$, it is trivial to

464 tell that its values vary across a small range between $0.12 \mathrm{~kg} / \mathrm{m}^{2}$ and $0.05 \mathrm{~kg} / \mathrm{m}^{2}$ and generally

465 decrease with the increasing rainfall intensity except for Gumbeel Ct. where $m_{0}^{*}$ has a smaller

466 value corresponding to the $40 \mathrm{~mm} / \mathrm{h}$ rain than $65 \mathrm{~mm} / \mathrm{h}$ and $86 \mathrm{~mm} / \mathrm{h}$. Compared with the

467 similar $m_{0}^{*}$ values on Lauder Ct. and Piccadilly P1., the corresponding values on Gumbeel Ct.

468 are greater. This may be explained by the different initial pollutant loads on the three roads and

469 the initial pollutant mass on Gumbeel Ct. was almost 3 times greater than other two sites, as

470 shown in Table 1. Therefore, the value of $m_{0}^{*}$ may be also related to the initial pollutant load

471 and further investigation will be needed in further research.

472

Table 3 The best-fitted values for $a_{d_{0}}$ and $m_{0}^{*}$.

\begin{tabular}{ccccccc}
\hline \multirow{2}{*}{ Rainfall intensity } & \multicolumn{2}{c}{ Lauder Ct. } & \multicolumn{2}{c}{ Gumbeel Ct. } & \multicolumn{2}{c}{ Piccadilly P1. } \\
\cline { 2 - 7 }$(\mathrm{mm} / \mathrm{h})$ & $a_{d_{0}}$ & $m_{0}^{*}$ & $a_{d_{0}}$ & $m_{0}^{*}$ & $a_{d_{0}}$ & $m_{0}^{*}$ \\
& $\left(\mathrm{~kg} / \mathrm{m}^{3}\right)$ & $\left(\mathrm{kg} / \mathrm{m}^{2}\right)$ & $\left(\mathrm{kg} / \mathrm{m}^{3}\right)$ & $\left(\mathrm{kg} / \mathrm{m}^{2}\right)$ & $\left(\mathrm{kg} / \mathrm{m}^{3}\right)$ & $\left(\mathrm{kg} / \mathrm{m}^{2}\right)$ \\
\hline 40 & 3800 & 0.09 & 3200 & 0.1 & 3500 & 0.09 \\
65 & 2300 & 0.08 & 2100 & 0.12 & 2200 & 0.09 \\
86 & 1600 & 0.08 & 1600 & 0.12 & 1600 & 0.09 \\
115 & 1600 & 0.06 & 1500 & 0.09 & 1600 & 0.08 \\
133 & 1500 & 0.06 & 1500 & 0.06 & 1500 & 0.05 \\
\hline
\end{tabular}

473 
(a) $40 \mathrm{~mm} / \mathrm{h}$

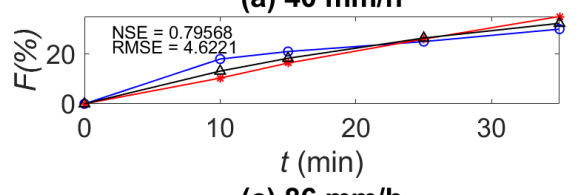

(c) $86 \mathrm{~mm} / \mathrm{h}$

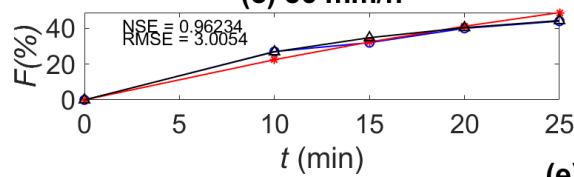

(b) $65 \mathrm{~mm} / \mathrm{h}$

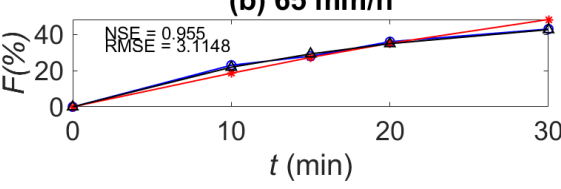

(d) $115 \mathrm{~mm} / \mathrm{h}$

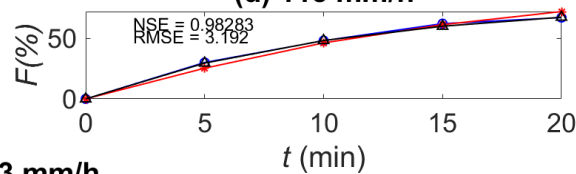

474

475

476

477

478

479

480

481

482

483

484

485

486

487

488

489

490

491

492

Fig. 12 Comparing the numerical results with measurements and empirical estimations obtained for different rainfall intensities on Lauder Ct.

The experiments are reproduced using the proposed particle-based stormwater quality model with the parameters as suggested in Tables 2 and 3. The results are plotted in Figs. 12-14, respectively for the three road sites, and compared with measurements of wash-off fraction $(F)$ and also the results obtained from the empirical equations as proposed by Egodawatta et al. (2007). All of the simulation results achieve an acceptable level of agreement with the measured data, indicating that the proposed model is capable of simulating the full-process of pollutant dynamics on urban surfaces after calibration. In particular for the results as shown in Fig. 13(c, e) and Fig. 14(b, c), the returned values of NSE generally show an increasing trend with increasing rainfall intensity. This may indicate that the proposed model performs better on heavy rains, which may be due to the adoption of median particle size to represent the whole range of pollutant particles. The light rains may not be efficient in detaching the sediments represented uniformly as a whole using the median particle size. But in reality, the light rains may still be able to efficiently detach the smaller sediments which are not well represented by the model (i.e. sediments smaller than the median particle size). This may subsequently lead to less accurate predictions as observed. Additionally, the model seems to overpredict the pollutants being washed off for short-duration rainfall events but underpredict for long-duration 
493 events on Gumbeel Ct., relative to the other two sites. But the predictions follow the same 494 trends as the results from the empirical equations, i.e. the observations from the Gumbeel Ct. 495 experiment do not agree as well with the equation results in comparison with the other two 496 sites, indicating abnormality in experimental measurements.

497 The runtimes for the five simulations for Lauder Ct., conducted on a Dell Latitude laptop 498 with a 4-core Intel i7-8650U CPU, are listed in Table 4, together with the event times. In all of 499 the simulations, a grid with $200 \times 150$ cells is adopted and $9 \times 10^{4}$ particles are used to 500 represent the initial pollutant load. Given the small simulation site, the computational time is 501 considered to be high and further improvement in computational efficiency is needed for large502 scale applications, which may be achieved through implementation of the entire coupled 503 modelling framework on GPUs.

Table 4 Computational time for Lauder Ct.

\begin{tabular}{ccc}
\hline $\begin{array}{c}\text { Rainfall intensity } \\
(\mathrm{mm} / \mathrm{h})\end{array}$ & Lauder Ct. \\
\hline 40 & 15 & Event Time (min) \\
65 & 13 & 35 \\
86 & 11 & 25 \\
115 & 10 & 25 \\
133 & 8 & 20 \\
\hline
\end{tabular}


(a) $40 \mathrm{~mm} / \mathrm{h}$

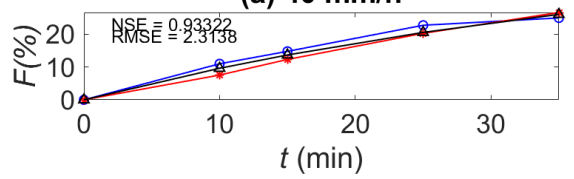

(c) $86 \mathrm{~mm} / \mathrm{h}$

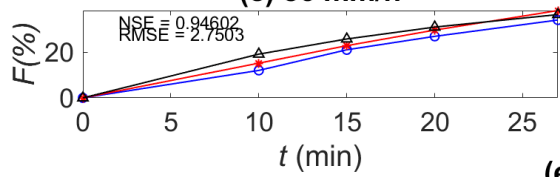

(b) $65 \mathrm{~mm} / \mathrm{h}$

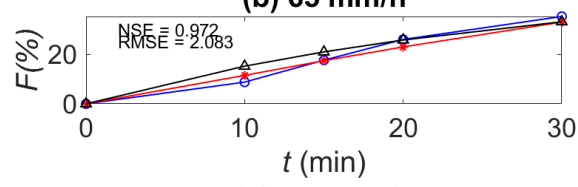

(d) $115 \mathrm{~mm} / \mathrm{h}$

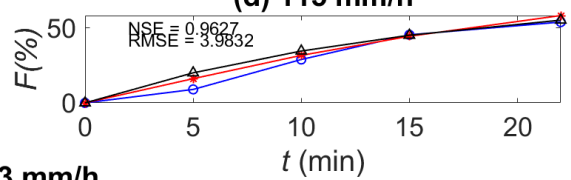

(e) $133 \mathrm{~mm} / \mathrm{h}$

506

507

508

509

510

511

512

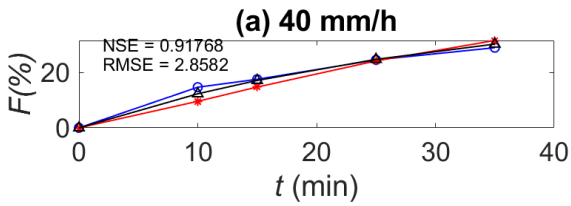

(c) $86 \mathrm{~mm} / \mathrm{h}$

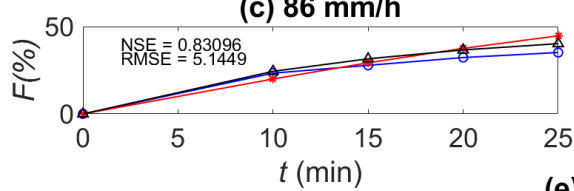

(e) $133 \mathrm{~mm} / \mathrm{h}$

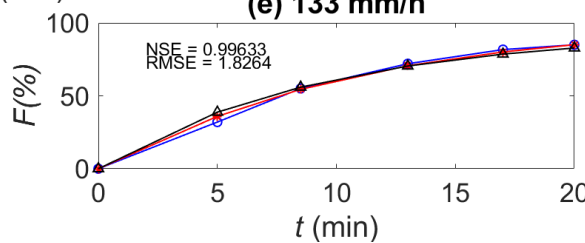
obtained for different rainfall intensities on Piccadilly P1. (b) $65 \mathrm{~mm} / \mathrm{h}$

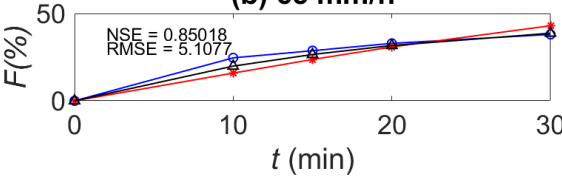

(d) $115 \mathrm{~mm} / \mathrm{h}$

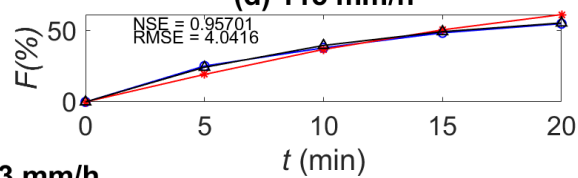

- O Observation Results

$\rightarrow$ - Simulation Results

$\triangle$ Equation Results

Fig. 14 Comparing the numerical results with measurements and empirical estimations

513 stormwater quality model may be also sensitive to other model settings, including e.g. grid

514 resolution and the number of particles being used to represent the pollutant loads. Herein, more

515 simulations are run to first test the convergence of simulation results against the number of

516 particles. Two typical rainfall intensities, $40 \mathrm{~mm} / \mathrm{h}$ and $133 \mathrm{~mm} / \mathrm{h}$, are selected and simulations

517 are run on all three road sites with the number of particles increasing from $0.1 \times 10^{4}$ to 
$51812 \times 10^{4}$. NSE and RMSE calculated against the wash-off fraction are used to assess model

519 performance and the results are presented in Fig. 15 and Fig. 16 respectively for the two

520 different rainfall intensities. Clearly, the numerical results appear to converge as the number of

521 particles increases and the simulation accuracy can no longer be further improved when the

522 number of particles moves beyond $3 \times 10^{4}$.

523

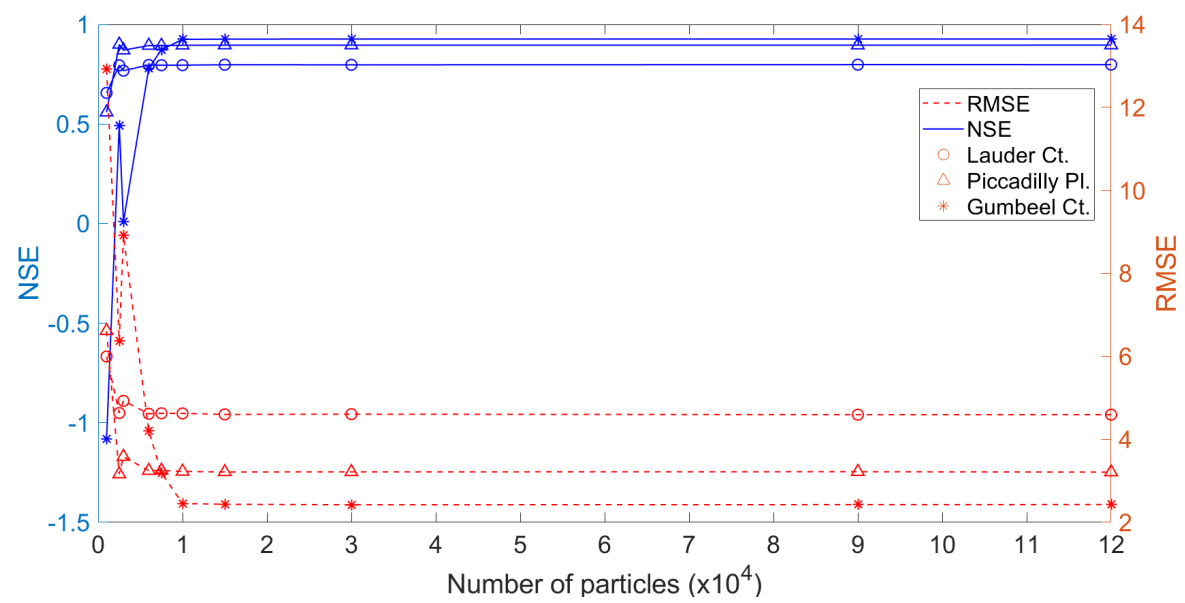

524 Fig. 15 Convergence test against the number of particles used to represent the initial pollutant loads on the three road sites using $40 \mathrm{~mm} / \mathrm{h}$ rainfall input.

526 To evaluate the sensitivity of the simulation results to the grid resolution, i.e. the number of 527 grid cells used to distribute pollutant particles during the wash-off process, further simulations 528 are conducted on the Piccadilly Pl. site using an example rainfall input of $133 \mathrm{~mm} / \mathrm{h}$. Based on 529 the results from the above convergence test against the number particles, $3 \times 10^{4}$ particles are 530 used and the number of grid cells varies from 12 to $12 \times 10^{4}$. Fig. 17 shows the simulation 531 results and it is clear that the results start to converge from 7,500 cells. 


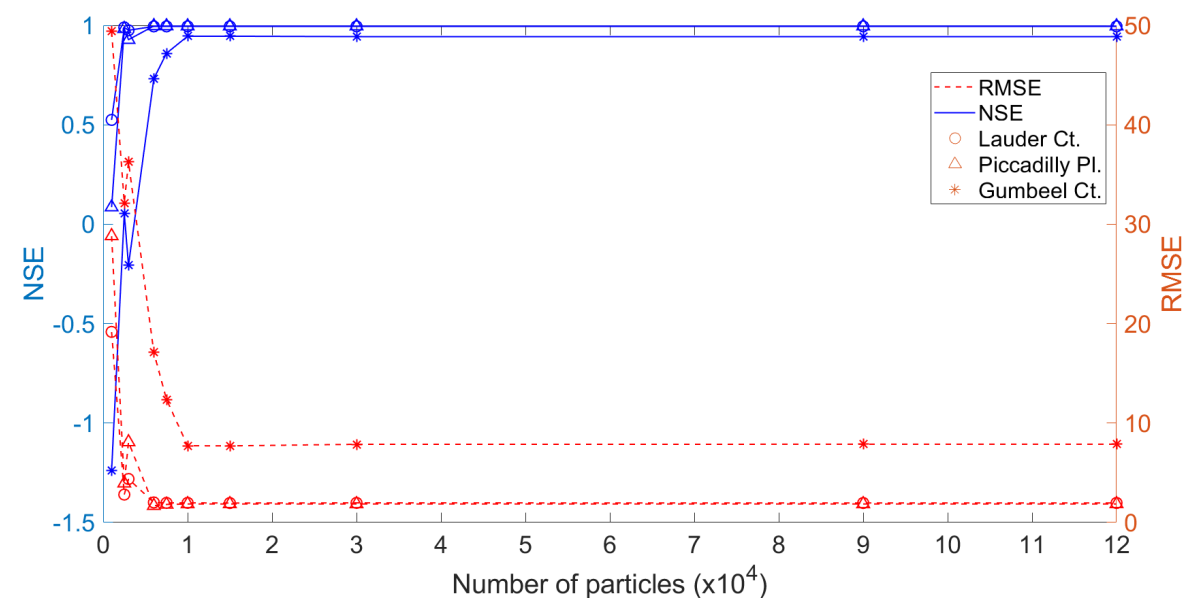

533 Fig. 16 Convergence test against the number of particles used to represent the initial pollutant loads on the three road sites using $133 \mathrm{~mm} / \mathrm{h}$ rainfall input.

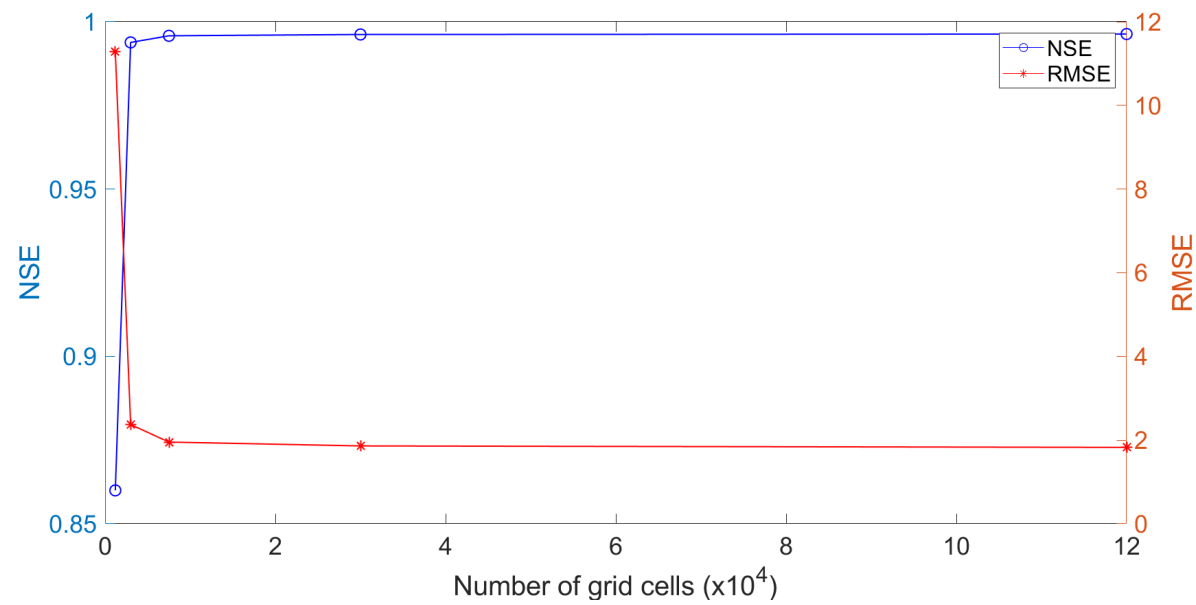

Fig. 17. Convergence test against the number of gird cells used to distribute pollutant particles.

539 Finally, the new coupled model is applied to an idealised V-shaped catchment to further 540 validate its capability in predicting runoff-driven pollutant dynamics on more complex 541 topographies involving SuDS features. As shown in Fig. 18, the two hillsides have a 542 topography chosen to be the same as the Lauder Ct. considered previously in section 3.3 and 543 are connected to a channel with a slope of 0.1 in the middle which is installed with a container 544 of $0.5 \mathrm{~m} \times 0.2 \mathrm{~m} \times 0.3 \mathrm{~m}$ to represent a detention pond and a $0.5 \mathrm{~m} \times 0.2 \mathrm{~m}$ infiltration area to 545 mimic a vegetated swale (represented by an infiltration rate of $3.61 \times 10^{-6} \mathrm{~m} / \mathrm{s}$ and a Manning 
coefficient of $0.1 \mathrm{~m} / \mathrm{s}^{1 / 3}$ ). A load of $9.3 \mathrm{~g}$ pollutants is uniformly distributed on the surface of each of the hillsides, but no pollutants are initially placed in the channel. Constant and uniform

548 rainfall of $133 \mathrm{~mm} / \mathrm{h}$ continuously falls on the whole catchment for $1200 \mathrm{~s}$ (i.e. $20 \mathrm{mins}$ ) from

549 the beginning. All boundaries are closed, except for the downstream channel outlet where open

550 boundary conditions are imposed. A uniform grid of $0.01 \mathrm{~m}$ resolution is used to discretise the 551 domain for the simulation.

552

553

554

555

556

557

558

559

560

561

562

563

564

565

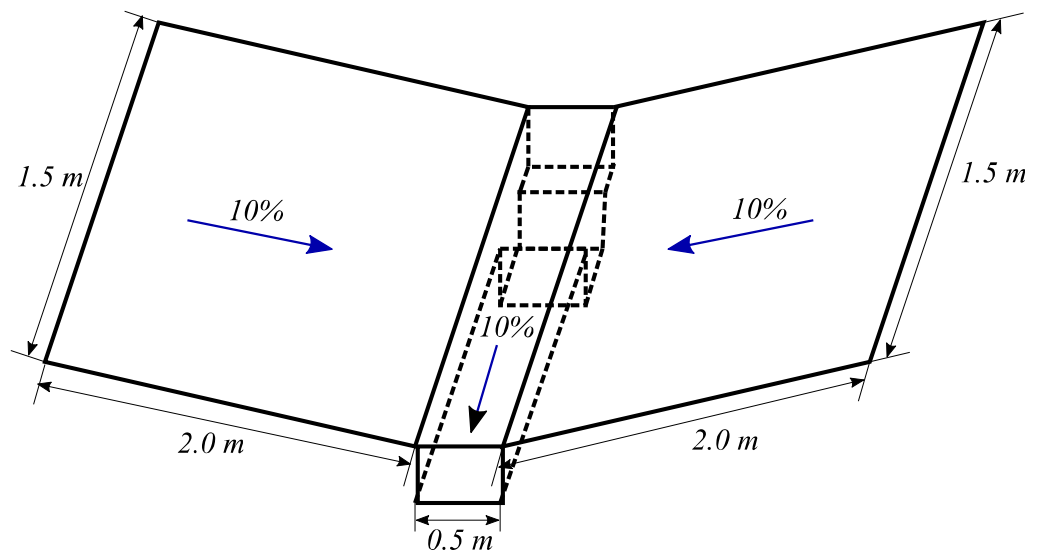

Fig. 18 Schematic of the V-shaped catchment with SuDS features.

Fig. 19(a) presents the hydrographs of water depth predicted by HiPIMS at the locations before (L1), inside (L2) and at the end of the detention pond (L3), inside (L4) and at the end of the swale (L5). The flow is intercepted by the pond and develops a larger water depth (L2) compared with the depths at other locations. The pond reaches its maximum capability after 449s; from then the water depth inside the pond (L2) stops growing and becomes steady. The water depths at the downstream locations (L3, L4, L5) show a small rise in sequence to reflect the release of water from the pond, as illustrated in Fig. 19(b). The difference of water depth between L3 and L4 indicates that the effect of higher bed friction (Manning coefficient) and infiltration in the swale has been correctly captured, which effectively reduces the overland flow at the downstream. Since the experimental settings for Lauder $\mathrm{Ct}$ are adopted in this test, the simulation results in terms of wash-off fraction should be consistent with the experimental measurements and this is confirmed in Fig. 20. 
(a)

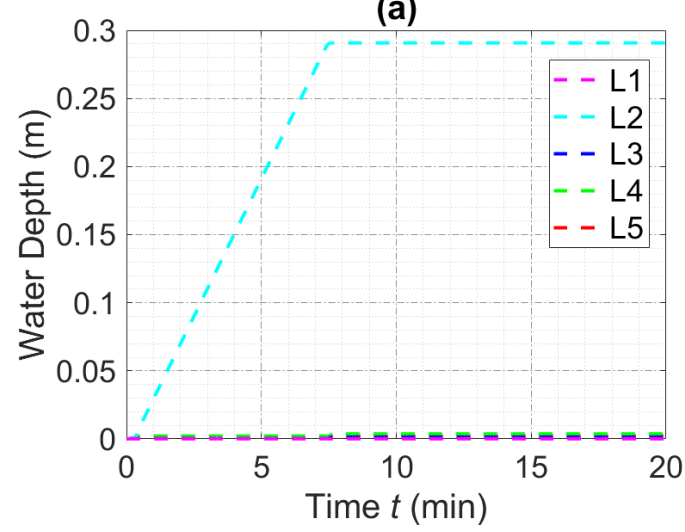

(b)

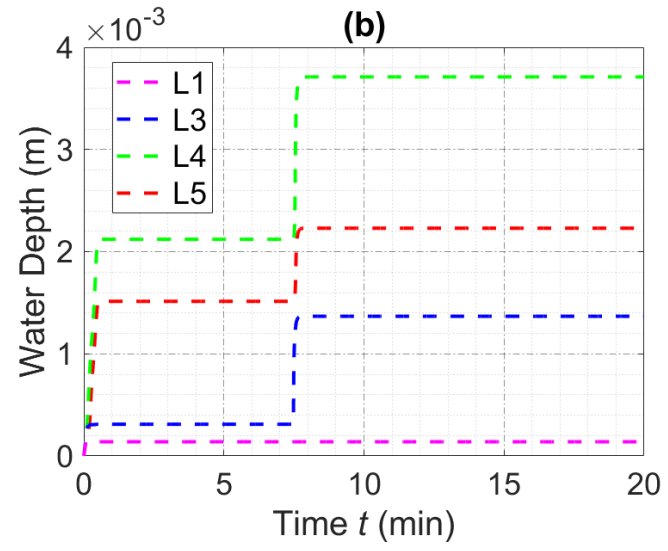

Fig. 21 Initial particle distribution at the hillsides.

573 To identify the sources of pollutants in the pond and the infiltration area, the initial 574 distribution of particles in the hillsides is divided into five regions marked in different colours, 575 as illustrated in Fig. 21, in which the location of the detention pond and the swale are also 576 indicated. Fig. 22 represents the particles located inside the detention pond and swale at 577 different output times. Apparently, due to its location, the detention pond only captures 
578 pollutants from regions $1 \& 2$, i.e. only red and blue particles are detected inside the spot, 579 during the whole rainfall event. The pollutants flow into the swale come from region 3 and 4 580 at the beginning. As time increases, pollutant particles from region $1 \& 2$ (red and blue particles) 581 start to 'leak' from the pond and travel to the swale. Since region 5 is downstream of both 582 SuDS features, the pollutants from this region are not captured by either of the features. To 583 quantify the composition of pollutant particles in the two SuDS features, percentages of 584 particles from different regions are calculated and plotted in Fig. 23 for (a) the detention pond 585 and (b) the swale. As confirmed in Fig. 23(a), the pond only captures particles from regions 1 $586 \& 2$ and the number of particles increases until approx. $t=10$ min to reach the pond's maximum 587 capability. After that, a small amount of pollutants starts to 'leak' from the pond and move 588 downstream to reach the swale area, which is reflected in the particle composition plot in Fig. 589 23(b) for the swale. For the swale, the number of particles (from regions $3 \& 4$ ) in the area 590 reaches its peak at the very earlier stage and starts to decrease afterwards. Since vegetation 591 interception and pollutant infiltration are not considered in the current model, the swale only 592 slows down the flow and also the movement of the particles but cannot intercepts any pollutants. 593 Therefore, the total amount of pollutants containing in the area are also smaller than those in 594 the pond at the corresponding moments. As a whole, the simulation results are as expected and 595 successful simulation of this test case demonstrates that the proposed modelling framework 596 can effectively identify the source and trace out the pathways of pollutants passing any specific 597 location. Such information will be very useful for the planning and design site-specific 598 controlling measures, i.e. SuDS, to more effectively manage urban surface pollutants. 

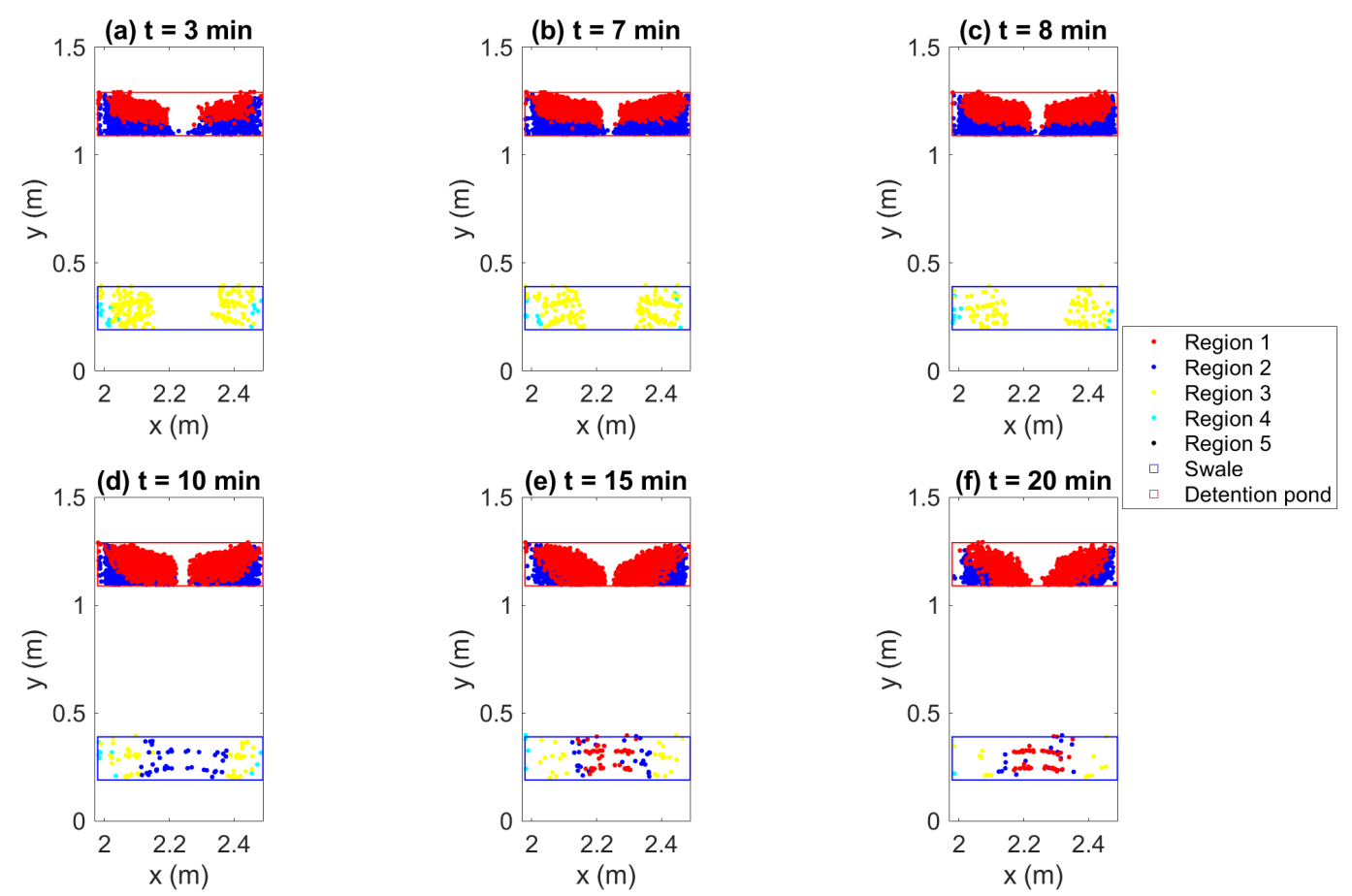

600 Fig. 22 Snapshots of particles located at the two SuDS features at different output times.

(a)

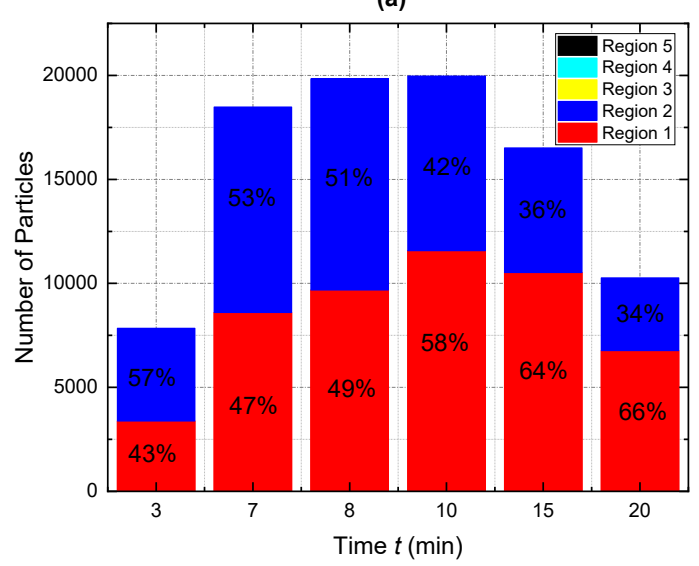

(b)

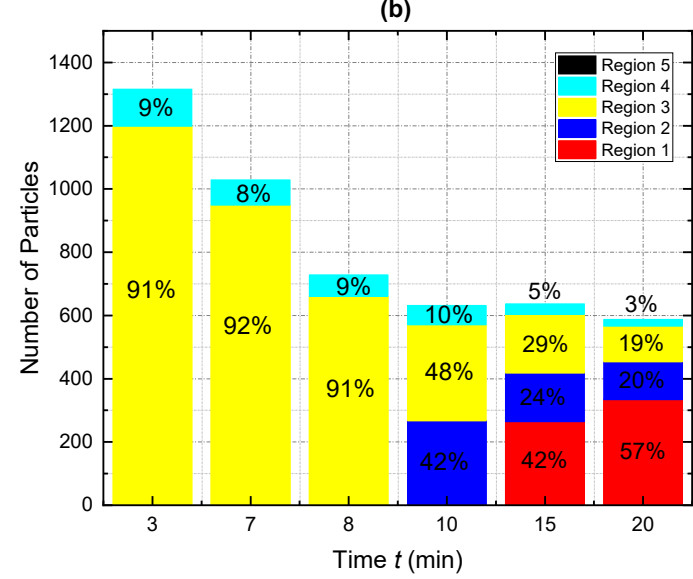

602 Fig. 23 Particle composition at the two SuDS features during the rainfall event: (a) Detention 603 pond; (b) Swale.

\section{4. Conclusions}

605 In this study, a new coupled hydrodynamic and particle-based water quality model is developed

606 to simulate the full-process dynamics of NPS particulate pollutants induced by rainfall in urban

607 areas, from pollutant detachment, transport to deposition. Based on a particle-based approach,

608 the model can effectively represent the physical processes of pollutant wash-off and transport

609 and trace out the trajectories or fates of individual particles to identify the pathways of NPS 
610 pollutants. To our best knowledge, this is the first time such a coupled modelling strategy is

611 reported to simulate the full-process dynamics of NPS pollutants in urban areas.

612 The new coupled model is successfully validated by simulating idealised and experimental 613 test cases with satisfactory results obtained, in comparison with analytical solutions or 614 experiment measurements. The current model is based on a coupled hydrodynamic and 615 particle-based modelling approach which can better depict the hydrodynamics of the rainfall 616 generated overland flow comparing with the traditional hydrological or simplified hydraulic 617 models. The use of the particle-based methods can effectively represent the Lagrangian 618 dynamics of the pollutant particles and trace out the trajectories of individual particles, which 619 can be subsequently used to derive the pathways of pollutants.

620 Whilst potentially providing a useful tool to improve the planning and design practice of 621 nature-based solutions (e.g. SuDS) to achieve multiple benefits in urban water management, 622 the current model still has a number of limitations that will benefit from further developments 623 in the future. Firstly, a single class size based on the median particle diameter is used to 624 represent pollutant loads in the model. The simulation results from the experiment test cases indicate that this may not be adequate to accurately depict the pollutant dynamics driven by 626 low-intensity rainfall and this is consistent with the conclusions reported by Hong et al. (2016a). 627 Secondly, further theoretical research is still needed to better understand the behaviours of certain model parameters, e.g. $a_{d_{0}}$ and $m_{0}^{*}$, for different applications. Furthermore, the model needs further improvement on computational efficiency, e.g. through implementing the entire 630 coupled modelling system on high-performance GPUs, for simulations involving large number 631 of particles over a large domain. Finally, the current NPS modelling system does not include modelling components to explicitly simulate different types of SuDS. Immediate future work will be to develop and couple compatible modelling strategies for different types of SuDS to support the planning and design of SuDS and assess their operation performance in practice. 
636 This work is partly supported by the ValBGI project (NE/S00288X/1) funded by the UK

637 Natural Environment Research Council (NERC).

638 References

639 Ahiablame, L.M., Engel, B.A., Chaubey, I., 2012. Effectiveness of low impact development

640 practices: Literature review and suggestions for future research. Water. Air. Soil Pollut.

641 223, 4253-4273. https://doi.org/10.1007/s11270-012-1189-2

642

Arthur, S., Krivtsov, V., Allen, D., 2019. Blue-green infrastructure - perspectives on water

643 quality benefits, C780b.

644

Bach, P.M., Rauch, W., Mikkelsen, P.S., McCarthy, D.T., Deletic, A., 2014. A critical review

645

646 of integrated urban water modelling - Urban drainage and beyond. Environ. Model. Softw. https://doi.org/10.1016/j.envsoft.2013.12.018

647

Baek, S.S., Choi, D.H., Jung, J.W., Lee, H.J., Lee, H., Yoon, K.S., Cho, K.H., 2015.

648

649

650

Optimizing low impact development (LID) for stormwater runoff treatment in urban area, Korea: Experimental and modeling approach. Water Res. 86, 122-131. https://doi.org/10.1016/j.watres.2015.08.038

Beuselinck, L., Hairsine, P.B., Govers, G., Poesen, J., 2002. Evaluating a single-class net deposition equation in overland flow conditions. Water Resour. Res. 38, 15-1-15-10. https://doi.org/10.1029/2001wr000248

Cheng, N.-S., 1997. Simplified settling velocity formula for sediment particles. J. Hydraul. 9429(1997)123:8(728) 

strategy to address diffuse water pollution from the built environment 26.

659 Egodawatta, P., Thomas, E., Goonetilleke, A., 2007. Mathematical interpretation of pollutant 660 wash-off from urban road surfaces using simulated rainfall. Water Res. 41, 3025-3031. $661 \quad$ https://doi.org/10.1016/j.watres.2007.03.037

662 Follett, E., Chamecki, M., Nepf, H., 2016. Evaluation of a random displacement model for 663 predicting particle escape from canopies using a simple eddy diffusivity model. Agric. 664 For. Meteorol. 224, 40-48. https://doi.org/10.1016/j.agrformet.2016.04.004

665 Gilbert, J.K., Clausen, J.C., 2006. Stormwater runoff quality and quantity from asphalt, 666 paver, and crushed stone driveways in Connecticut. Water Res. 40, 826-832.

667 https://doi.org/10.1016/j.watres.2005.12.006

Gomez-Gesteira, M., Montero, P., Prego, R., Taboada, J.J., Leitao, P., Ruiz-Villarreal, M., 669 Neves, R., Perez-Villar, V., 1999. A two-dimensional particle tracking model for pollution dispersion in A Coruna and Vigo Rias (NW Spain). Oceanol. Acta 22, $167-$

Hairsine, P.B., Rose, C.W., 1992a. Modeling water erosion due to overland flow using physical principles: 2. Rill flow. Water Resour. Res. 28, 245-250. https://doi.org/10.1029/91WR02381

Hairsine, P.B., Rose, C.W., 1992b. Modeling water erosion due to overland flow using physical principles: 1. Sheet flow. Water Resour. Res. 28, 237-243.

Heng, B.C.P., Sander, G.C., Armstrong, A., Quinton, J.N., Chandler, J.H., Scott, C.F., 2011. Modeling the dynamics of soil erosion and size-selective sediment transport over nonuniform topography in flume-scale experiments. Water Resour. Res. 47. 
682

683

684

685

686

687

688

689

690

691

692

693

694

695

696

697

698

699

700

701

702

703

Hong, Y., Bonhomme, C., Le, M.H., Chebbo, G., 2016a. A new approach of monitoring and physically-based modelling to investigate urban wash-off process on a road catchment near Paris. Water Res. 102, 96-108. https://doi.org/10.1016/j.watres.2016.06.027

Hong, Y., Bonhomme, C., Le, M.H., Chebbo, G., 2016b. New insights into the urban washoff process with detailed physical modelling. Sci. Total Environ. 573, 924-936. https://doi.org/10.1016/j.scitotenv.2016.08.193

Hong, Y., Liao, Q., Bonhomme, C., Chebbo, G., 2019. Physically-based urban stormwater quality modelling: An efficient approach for calibration and sensitivity analysis. J. Environ. Manage. 246, 462-471. https://doi.org/10.1016/j.jenvman.2019.06.003

Huai, W., Yang, L., Wang, W.J., Guo, Y., Wang, T., Cheng, Y. guang, 2019. Predicting the vertical low suspended sediment concentration in vegetated flow using a random displacement model. J. Hydrol. 578. https://doi.org/10.1016/j.jhydrol.2019.124101

Jomaa, S., Barry, D.A., Brovelli, A., Sander, G.C., Parlange, J.Y., Heng, B.C.P., Tromp-van Meerveld, H.J., 2010. Effect of raindrop splash and transversal width on soil erosion: Laboratory flume experiments and analysis with the Hairsine-Rose model. J. Hydrol. 395, 117-132. https://doi.org/10.1016/j.jhydrol.2010.10.021

Liang, Q., Smith, L.S., 2015. A High-Performance Integrated hydrodynamic Modelling System for urban flood simulations. J. Hydroinformatics 17, 518-533. https://doi.org/10.2166/hydro.2015.029

Liang, Q., Taylor, P.H., Borthwick, A.G.L., 2007. Particle mixing and reactive front motion in unsteady open shallow flow - Modelled using singular value decomposition. Comput. Fluids 36, 248-258. https://doi.org/10.1016/j.compfluid.2006.01.018 
Mutchler, C.K., Hansen, L.M., 1970. Splash of a waterdrop at terminal velocity. Science

Obropta, C.C., Kardos, J.S., 2007. Review of Urban Stormwater Quality Models: Deterministic, Stochastic, and Hybrid Approaches1. JAWRA J. Am. Water Resour. Assoc. 43, 1508-1523. https://doi.org/10.1111/j.1752-1688.2007.00124.x

709

710

Olascoaga, M.J., Beron-Vera, F.J., Haller, G., Triñanes, J., Iskandarani, M., Coelho, E.F., Haus, B.K., Huntley, H.S., Jacobs, G., Kirwan, A.D., Lipphardt, B.L., Özgökmen, T.M., H. M. Reniers, A.J., Valle-Levinson, A., 2013. Drifter motion in the Gulf of Mexico constrained by altimetric Lagrangian coherent structures. Geophys. Res. Lett. 40, 61716175. https://doi.org/10.1002/2013GL058624

Paresi, M., Melchiorri, M., Siragusa, A., Kemper, T., 2016. Atlas of the Human Planet. Mapping Human Presence on Earth with the Global Human Settlement Layer. https://doi.org/10.2788/889483

Proffitt, A.P.B., Rose, C.W., Hairsine, P.B., 1991. Rainfall Detachment and Deposition: Experiments with Low Slopes and Significant Water Depths. Soil Sci. Soc. Am. J. 55, 325. https://doi.org/10.2136/sssaj1991.03615995005500020004x

Rossman, L.A., Huber, W.C., 2016. Storm Water Management Model Reference Manual Volume III - Water Quality. United States Environ. Prot. Agency III, 235. https://doi.org/EPA/600/R-15/162 |

Sage, J., Bonhomme, C., Al Ali, S., Gromaire, M.C., 2015. Performance assessment of a commonly used "accumulation and wash-off" model from long-term continuous road runoff turbidity measurements. Water Res. 78, 47-59. https://doi.org/10.1016/j.watres.2015.03.030 
Sämann, R., Graf, T., Neuweiler, I., 2019. Modeling of contaminant transport during an urban pluvial flood event - The importance of surface flow. J. Hydrol. 568, 301-310. https://doi.org/10.1016/j.jhydrol.2018.10.002

Shaw, S.B., Parlange, J.Y., Lebowitz, M., Todd Walter, M., 2009. Accounting for surface roughness in a physically-based urban wash-off model. J. Hydrol. 367, 79-85. https://doi.org/10.1016/j.jhydrol.2009.01.004

Shaw, S.B., Walter, M.T., Steenhuis, T.S., 2006. A physical model of particulate wash-off from rough impervious surfaces. J. Hydrol. 327, 618-626. https://doi.org/10.1016/j.jhydrol.2006.01.024

Tsihrintzis, V.A., Hamid, R., 1997. Modeling and management of urban stormwater runoff quality: A review. Water Resour. Manag. https://doi.org/10.1023/a:1007903817943

Weitbrecht, V., Uijttewaal, W., Jirka, G.H., 2003. 2-D Particle Tracking to Determine Transport Characteristics in Rivers with Dead Zones. Shallow flows 1-8.

Xia, X., Liang, Q., Ming, X., 2019. A full-scale fluvial flood modelling framework based on a high-performance integrated hydrodynamic modelling system (HiPIMS). Adv. Water Resour. 132. https://doi.org/10.1016/j.advwatres.2019.103392

Zhang, L., Liang, Q., Wang, Y., Yin, J., 2015. A robust coupled model for solute transport driven by severe flow conditions. J. Hydro-Environment Res. 9, 49-60. https://doi.org/10.1016/j.jher.2014.04.005 